\title{
Long noncoding RNA NEXN-AS1 mitigates atherosclerosis by regulating the actin-binding protein NEXN
}

\author{
Yan-Wei Hu, ${ }^{1}$ Feng-Xia Guo, ${ }^{1}$ Yuan-Jun Xu, ${ }^{1}$ Pan Li, ${ }^{1}$ Zhi-Feng Lu, ${ }^{1}$ David G. McVey, ${ }^{2}$ Lei Zheng, ${ }^{1}$ Qian Wang, ${ }^{1}$ John H. Ye, ${ }^{3}$ \\ Chun-Min Kang, ${ }^{1}$ Shao-Guo Wu, ${ }^{4}$ Jing-Jing Zhao, ${ }^{1}$ Xin Ma, ${ }^{5}$ Zhen Yang, ${ }^{6}$ Fu-Chun Fang, ${ }^{7}$ Yu-Rong Qiu, ${ }^{1}$ Bang-Ming Xu, ${ }^{1}$ \\ Lei Xiao, ${ }^{1}$ Qian Wu, ${ }^{1}$ Li-Mei Wu, ${ }^{3}$ Li Ding, ${ }^{1}$ Tom R. Webb, ${ }^{2}$ Nilesh J. Samani, ${ }^{2}$ and Shu Ye ${ }^{2,8}$ \\ 'Laboratory Medicine Center, Nanfang Hospital, Southern Medical University, Guangzhou, China. ²Department of Cardiovascular Sciences, and NIHR Leicester Biomedical Research Centre, University of \\ Leicester, Leicester, United Kingdom. ${ }^{3}$ University Hospitals of Leicester NHS Trust, Leicester, United Kingdom. Department of Clinical Laboratory, Guangzhou Twelfth People's Hospital, Guangzhou, China. \\ ${ }^{5}$ Department of Anesthesiology, Nanfang Hospital, Southern Medical University, Guangzhou, China. ${ }^{6}$ Department of Cardiology, The First Affiliated Hospital, Sun Yat-Sen University, Guangzhou, China. \\ ${ }^{7}$ Department of Stomatology, Nanfang Hospital, Southern Medical University, Guangzhou, China. ${ }^{8}$ Shantou University Medical College, Shantou, China.
}

\begin{abstract}
Noncoding RNAs are emerging as important players in gene regulation and disease pathogeneses. Here, we show that a previously uncharacterized long noncoding RNA, nexilin F-actin binding protein antisense RNA 1 (NEXN-AS1), modulates the expression of the actin-binding protein NEXN and that NEXN exerts a protective role against atherosclerosis. An expression microarray analysis showed that the expression of both NEXN-AS1 and NEXN was reduced in human atherosclerotic plaques. In vitro experiments revealed that NEXN-AS1 interacted with the chromatin remodeler BAZ1A and the $5^{\prime}$ flanking region of the NEXN gene and that it also upregulated NEXN expression. Augmentation of NEXN-AS1 expression inhibited TLR4 oligomerization and NF- $\kappa B$ activity, downregulated the expression of adhesion molecules and inflammatory cytokines by endothelial cells, and suppressed monocyte adhesion to endothelial cells. These inhibitory effects of NEXN-AS1 were abolished by knockdown of NEXN. In vivo experiments using ApoE-knockout mice fed a Western high-fat diet demonstrated that NEXN deficiency promoted atherosclerosis and increased macrophage abundance in atherosclerotic lesions, with heightened expression of adhesion molecules and inflammatory cytokines, whereas augmented NEXN expression deterred atherosclerosis. Patients with coronary artery disease were found to have lower blood NEXN levels than healthy individuals. These results indicate that NEXN-AS1 and NEXN represent potential therapeutic targets in atherosclerosis-related diseases.
\end{abstract}

\section{Introduction}

Atherosclerosis underlies the vast majority of coronary artery disease (CAD) and contributes to the development of many other cardiovascular disorders. The pathogenesis of atherosclerosis is complex and still incompletely understood. It is known that early events that lead to the development of the atherosclerotic plaque include endothelial activation, with increased production of chemokines and cytokines, and recruitment of circulating leukocytes to the affected arterial intima together with local deposition of lipids and accumulation of vascular smooth muscle cells (VSMCs) and extracellular matrix proteins $(1,2)$. At an advanced stage of the disease, atherosclerotic plaques with a high abundance of monocyte-derived macrophages, but low content of VSMCs and collagens, are prone to rupture, which can trigger ischemic events, such as myocardial infarction (3). These processes involve numerous regulatory and effector molecules and their interplay. Although a considerable number of such molecules have been shown to participate in the pathogenesis of atherosclerosis, it is likely that more are still yet to be uncovered.

Conflict of interest: The authors have declared that no conflict of interest exists. License: Copyright 2019, American Society for Clinical Investigation.

Submitted: October 23, 2017; Accepted: December 18, 2018.

Reference information: / Clin Invest. 2019;129(3):1115-1128.

https://doi.org/10.1172/JCI98230.
The Human Genome Project has shown that there are approximately 20,000 protein-coding genes in the human nuclear genome; this number is much smaller than the 100,000 previously estimated $(4,5)$. These protein-coding genes occupy only about $1 \%$ of the human nuclear genome, while most of the remainder of the genome was thought to be of "junk" DNA without important biological function (5). However, more recently, the Encyclopedia of DNA Elements (ENCODE) consortium has discovered that there exists a very large number of noncoding RNA species, some of which are encoded by part of the genome previously considered to be junk DNA. There is accumulating evidence indicating that a significant proportion of these noncoding RNA species have important biological functions $(6,7)$. Noncoding RNAs can be divided into small noncoding RNAs ( $<200$ nucleotides) and longer noncoding RNAs (>200 nucleotides), respectively; the former class consists of microRNAs, transfer RNAs, and small nucleolar RNAs, while the latter class includes ribosomal RNAs, natural antisense transcripts, and other long noncoding RNAs (lncRNAs) (7). Recent studies have identified a number of microRNAs and lncRNAs that play important roles in regulating gene expression and are associated with cardiovascular diseases (7). As this is a research area in its infancy, it is likely that there are still more functional noncoding RNAs with involvement in cardiovascular disease pathogenesis yet to be identified. 

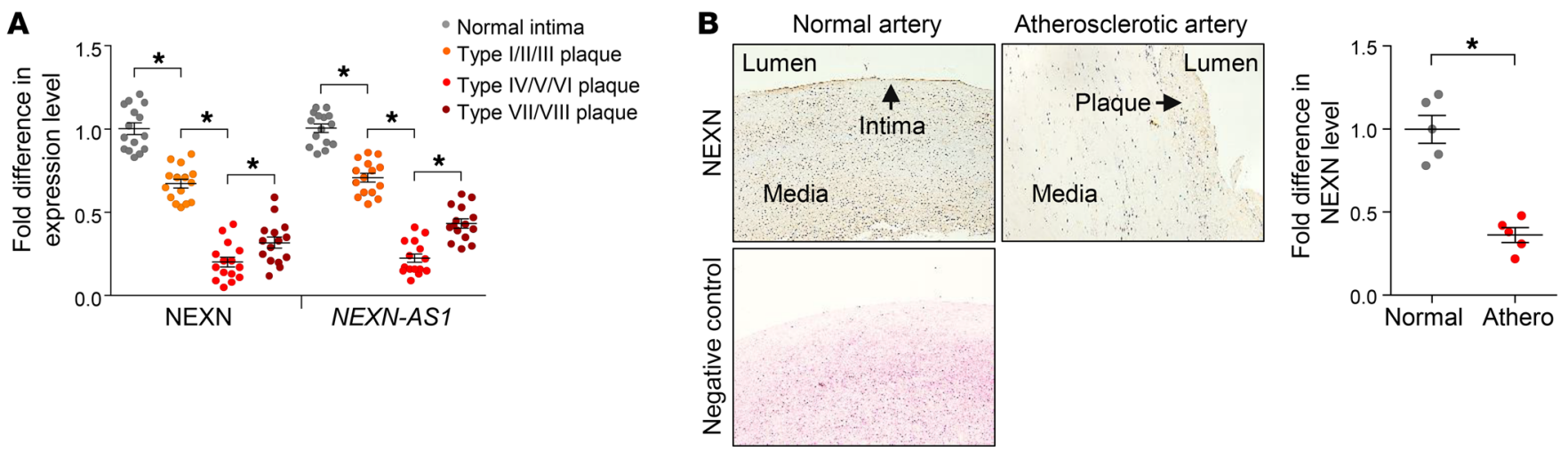

C

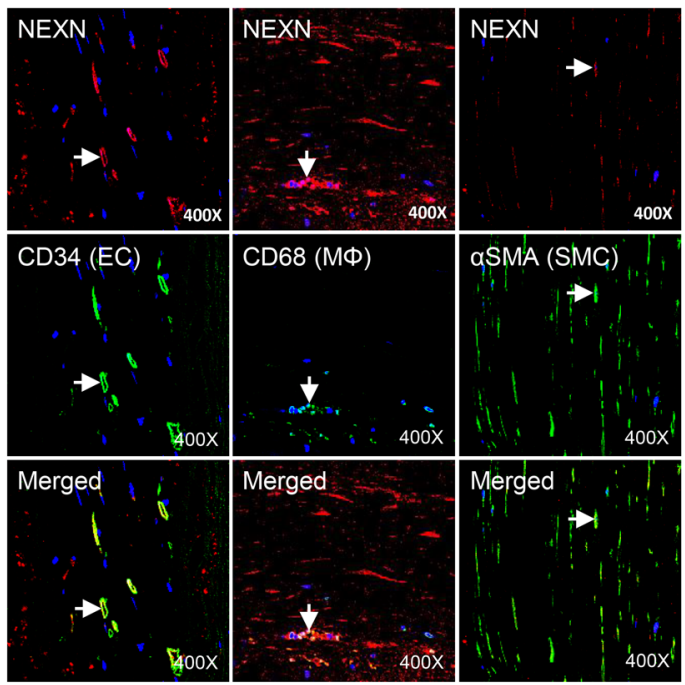

D

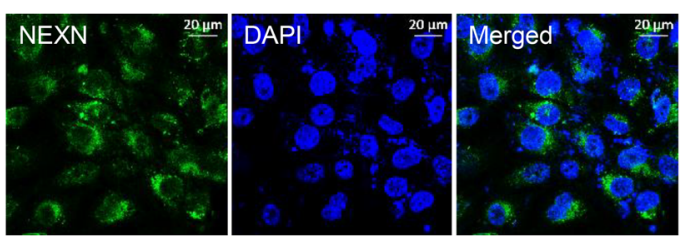

E

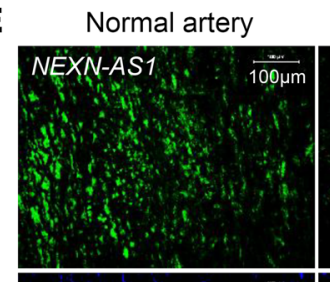

Atherosclerotic artery
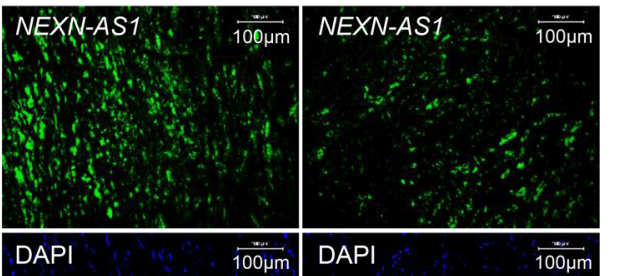

$\underset{100 \mu \mathrm{m}}{\stackrel{m a t}{2}}$
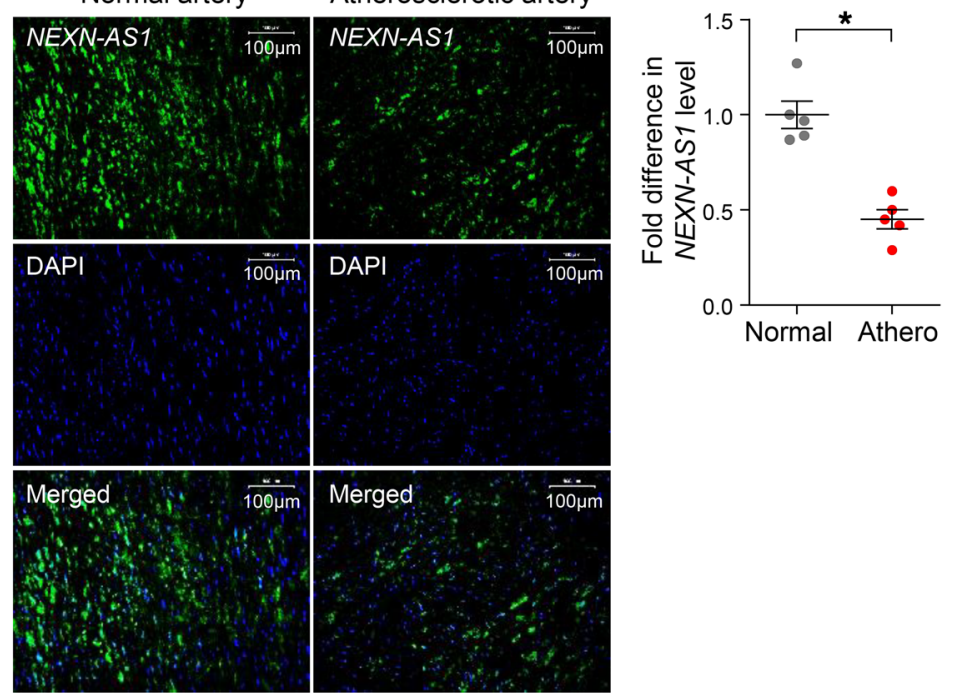

$\mathbf{F}$

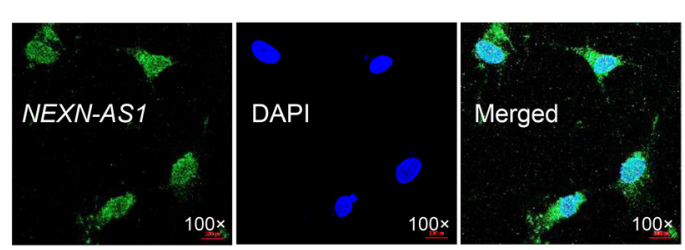

Figure 1. Expression of NEXN and NEXN-AS1 in atherosclerotic plaques. (A) NEXN and NEXN-AS1 expression levels in human normal and atherosclerotic arteries, quantified by RT-PCR. The graph shows fold differences in mean \pm SD NEXN and NEXN-AS1 RNA levels. $n=5$ subjects in each group, each assayed in triplicate. ${ }^{*} P<0.05$, ANOVA with post hoc analysis and Bonferroni's correction. (B) NEXN protein in human normal and atherosclerotic arteries, detected by immunohistochemistry. Left: representative images of immunohistochemical staining of NEXN (stained brown) in normal and atherosclerotic arterial tissues and image of negative control without the primary antibody (anti-NEXN antibody). Original magnification, $\times 200$. Right: fold difference in mean \pm SD NEXN level. $n=5$ subjects in each group. ${ }^{*} P<0.05, t$ test. Athero, atherosclerotic. (C) Presence of NEXN in endothelial cells (EC) in intraplaque neovessels, macrophages, and VSMCs in human atherosclerotic plaques, detected by double immunostaining with the use of antibodies against NEXN, the EC marker CD34, the macrophages marker CD68, and the VSMC marker $\alpha$ SMA, respectively. Original magnification, $\times 400$. (D) Intracellular location of NEXN in cultured human vascular endothelial cells, determined by immunofluorescence microscopy. NEXN was stained green using an anti-NEXN antibody and the nucleus stained blue with DAPI. Scale bars: $20 \mu \mathrm{m}$. (E) NEXN-AS1 RNA in human normal and atherosclerotic arteries, detected by FISH. Left: representative FISH images. Scale bars: $100 \mu \mathrm{m}$. Right: fold difference in mean \pm SD NEXN-AS1 levels. $n=5$ subjects in each group. ${ }^{*} P<0.05, t$ test. (F) Presence of NEXN-AS1 in both the cytoplasm and nucleus in cultured human vascular endothelial cells, detected by FISH. NEXN-AS1 was stained green using an RNA probe, and the nucleus was stained blue with DAPI. Original magnification, $\times 400$.

Nexilin F-actin-binding protein (NEXN), also called Nexilin, is an actin-binding protein that is thought to function in cell adhesion and migration. Mutations in the NEXN gene have been associated with dilated cardiomyopathy and hypertrophic cardiomyopathy in humans $(8,9)$. Additionally, a recent study reported an association between variation in the NEXN gene and susceptibility to CAD in Han Chinese (10), providing evidence of a link between the NEXN gene and CAD, although there is lack of evidence for such an association in other populations. Furthermore, the authors showed that NEXN inhibited balloon injury-induced neointima formation in a rat model (10). 
We report here the findings from a study of a previously uncharacterized lncRNA, NEXN antisense RNA 1 (NEXN-AS1), and its cognate gene, NEXN. In brief, we found that (a) both $N E X N-A S 1$ and NEXN have decreased expression levels in human atherosclerotic plaques; (b) $N E X N-A S 1$ interacts with the chromatin remodeler BAZ1A and upregulates NEXN gene expression; (c) $N E X N-A S 1$ and NEXN inhibit endothelial activation and monocyte recruitment; (d) NEXN deficiency results in increased atherosclerosis, whereas NEXN overexpression deters atherosclerosis, in an in vivo experimental model; and (e) patients with CAD have lower circulating NEXN levels.

\section{Results}

Reduced expression of NEXN-AS1 and NEXN in human atherosclerotic plaques. To identify differentially expressed genes in human atherosclerotic plaques, we performed an expression microarray analysis on aortic atherosclerotic plaque cap specimens (from 3 patients) and healthy aortic tissues (from 3 individuals) using the Arraystar LncRNA Expression Microarray, version 3.0, which contained probes for 24,420 protein coding transcripts and 24,748 lncRNAs. The analysis identified a number of differentially expressed genes (Supplemental Tables 1 and 2; supplemental material available online with this article; https://doi.org/10.1172/JCI98230DS1), including the protein-coding gene NEXN and a cognate lncRNA gene, NEXN-AS1, both of which showed significantly lower RNA levels in atherosclerotic plaques than in healthy aortic tissues $(P=$ $6.12 \times 10^{-4}$ and $P=8.91 \times 10^{-8}$, respectively). A recent study reported an association between variation in the NEXN gene and susceptibility to CAD and showed that adenovirus-mediated NEXN overexpression inhibited balloon injury-induced neointima formation in a rat model (10). It raises the possibility that NEXN might also play a role in de novo atherosclerosis, which warrants investigation. Therefore, among the differentially expressed genes identified by the abovementioned microarray analysis, we chose to focus on $N E X N$ and $N E X N-A S 1$ in our present study.

A quantitative reverse-transcriptase PCR (RT-PCR) analysis of samples from additional subjects confirmed that the RNA levels of both $N E X N$ and $N E X N-A S 1$ were lower in atherosclerotic plaques (of either the carotid artery or abdominal aorta, from 15 patients) than in healthy arterial intima tissues (from 5 individuals) and additionally showed that their levels were lower in advanced atherosclerotic plaques (American Heart Association classification types IV-VIII [ref. 11], from 10 patients) than in early plaques (types I-III [ref. 11], from 5 patients) and lower in advanced vulnerable plaques (types IV, V, and VI [ref. 11], from 5 patients) than in advanced stable plaques (types VII and VIII [ref. 11], from 5 patients) (Figure 1A).

Consistent with the above quantitative RT-PCR results, immunohistochemistry showed that atherosclerotic arteries had lower NEXN protein levels, as compared with normal arteries (Figure 1B). Double immunostaining of NEXN along with the endothelial cell marker CD34, the macrophage marker CD68, and the VSMC marker aSMA, respectively, showed that, in atherosclerotic plaques, these 3 cell types (endothelial cells, macrophages, and VSMCs) all expressed NEXN (Figure 1C). An immunofluorescence confocal microscopy examination of cultured vascular endothelial cells showed that NEXN was present primarily in the cytoplasm (Figure 1D).
FISH demonstrated that atherosclerotic plaques had lower $N E X N$-AS1 RNA levels (Figure 1E). An analysis of in vitro cultures of human umbilical vein endothelial cells (HUVECs), monocytes (THP-1 cells), and VSMCs showed that these 3 types of cells all expressed $N E X N-A S 1$, with an estimate of 9 copies of the $N E X N$ AS1 RNA per vascular endothelial cell, 58 copies per monocyte, and 3 copies per VSMC. An immunofluorescence confocal microscopy examination of cultured vascular endothelial cells detected the presence of NEXN-AS1 RNA in both the cytoplasm and nucleus (Figure $1 \mathrm{~F}$ ).

NEXN-AS1 upregulates NEXN expression. The NEXN and $N E X N-A S 1$ genes are both located on chromosome 1, with a proportion of NEXN-AS1 overlapping with the $5^{\prime}$ portion of NEXN (Supplemental Figure 1). Since some other lncRNAs have been reported to modulate the expression of nearby protein-coding genes $(6,7)$, we wondered whether the lncRNA $N E X N-A S 1$ has an effect on NEXN expression. To address this question, we transfected cultured vascular endothelial cells with a lentivirus (Supplemental Figure 2) to overexpress NEXN-AS1 in-trans and found that $N E X N-A S 1$ overexpression led to an increase in NEXN mRNA and NEXN protein levels (Figure 2, A and B), suggesting that $N E X N-A S 1$ upregulates $N E X N$ expression. In support, shRNAmediated NEXN-AS1 knockdown in cultured vascular endothelial cells resulted in a decrease in NEXN levels (Figure 2C).

To investigate the molecular mechanism through which NEXN-AS1 modulates NEXN expression, we performed analysis by chromatin isolation by RNA purification (ChIRP), a method that allows unbiased screening of RNA-bound DNA and proteins. The analysis showed that chromatin samples pulled down by NEXN-AS1 RNA probes (Supplemental Figure 3) harbored the $N E X N$ gene $5^{\prime}$ flanking region (Figure $2 \mathrm{D}$ ), suggesting an interaction between lncRNA NEXN-AS1 and the 5' flanking region of the $N E X N$ gene. To investigate whether NEXN-AS1 affects the activity of the $N E X N 5^{\prime}$ flanking region, we performed a reporter assay in which we transfected cultured vascular endothelial cells with a plasmid containing the NEXN gene 5 ' flanking sequence (nucleotide positions -1245 bp to +297 bp relative to the transcription start site) and a luciferase reporter gene, with or without cotransfection of a lentivirus to overexpress $N E X N-A S 1$, followed by measurement of luciferase activity. The assay showed that NEXN-AS1 overexpression enhanced the ability of the NEXN gene 5 ' flanking sequence to drive the expression of the luciferase reporter gene (Figure 2E). In a parallel assay that was the same as above, except the nucleotides between $+1 \mathrm{bp}$ and $+245 \mathrm{bp}$ relative to the NEXN transcription start site in the NEXN gene 5 ' flanking sequence were deleted from the plasmid (Supplemental Figure 3), NEXN-AS1 overexpression no longer increased the transcriptional activity of the NEXN gene 5' flanking sequence to drive luciferase reporter gene expression (Figure 2E), suggesting that the region between +1 bp and +245 bp relative to the $N E X N$ transcription start site is essential for NEXN-AS1 regulation of NEXN expression.

A protein mass spectrometric analysis of the chromatin complex captured by the NEXN-AS1 RNA probes in the abovementioned ChIRP experiment detected the presence of several histone proteins and other DNA/RNA-binding proteins (Supplemental Table 3). Among these proteins, BAZ1A (also known as ATP-utilizing chromatin assembly and remodeling factor $\underline{1}$ [ACF1]) is of par- 
A

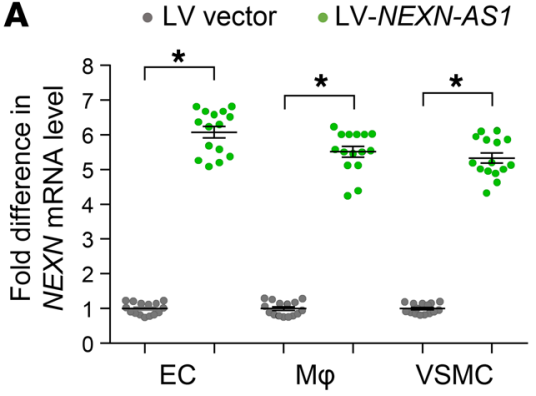

C

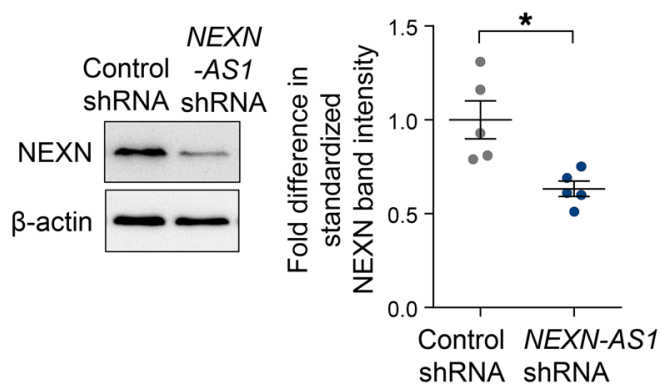

B

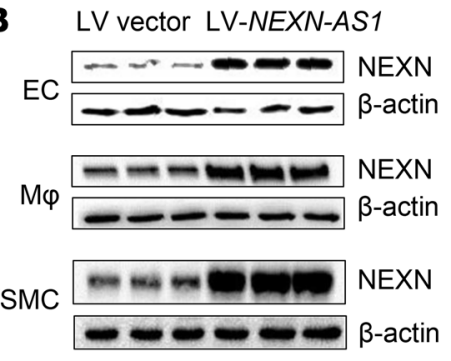

D

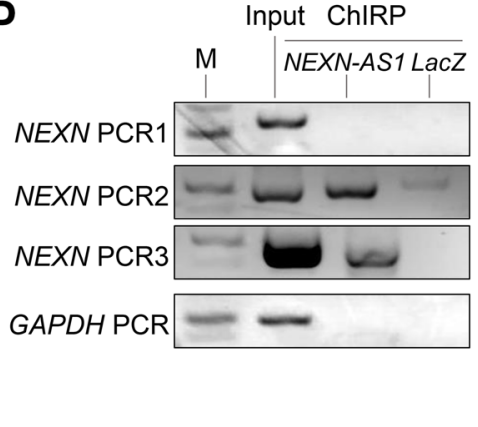

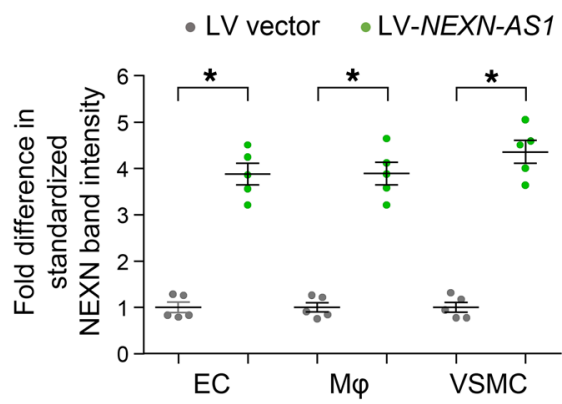

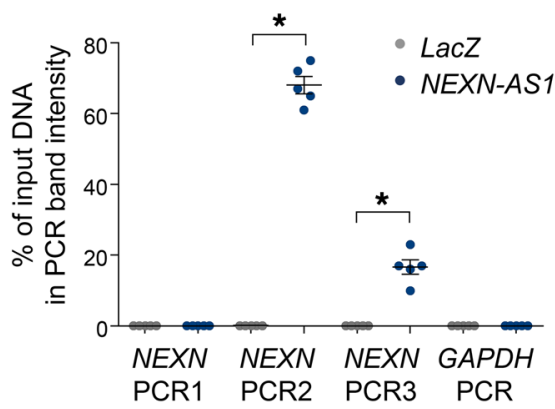

$\mathbf{E}$

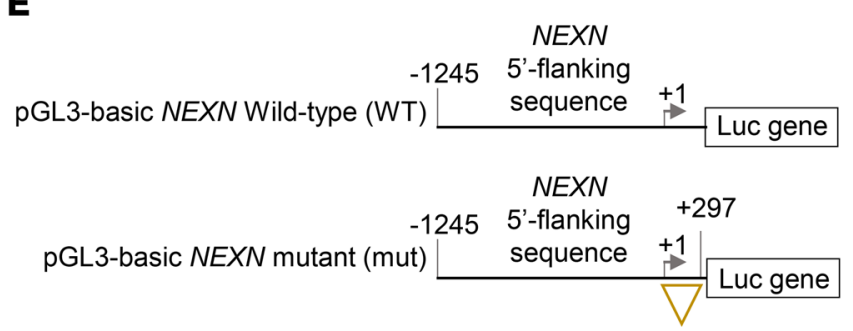

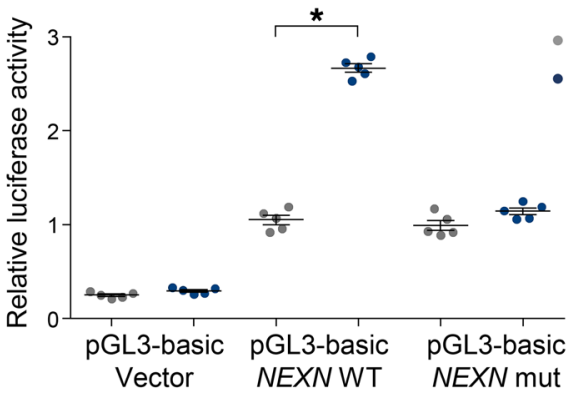

Figure 2. NEXN-AS1 upregulates NEXN expression. (A) Cultured human vascular endothelial cells, macrophages, and VSMCs were transfected with either an NEXN-AS1-expressing lentivirus (LV-NEXN-AS1) or the lentivirus vector (LV vector) as a control, followed by quantitative RT-PCR analysis of NEXN mRNA levels. The column chart shows fold difference in mean \pm SD NEXN mRNA levels in 5 independent experiments with each experiment done in triplicate. ${ }^{*} P<0.05, t$ test. (B) Cultured human ECs, macrophages, and VSMCs were transfected with either LV-NEXN-AS1 or LV vector, followed by immunoblot analysis of the NEXN protein. Left: representative immunoblot images; right: fold differences in mean \pm SD NEXN band intensity after standardization against the band intensity of the housekeeping protein $\beta$-actin in 5 independent experiments. ${ }^{*} P<0.05, t$ test. (C) Cultured human vascular endothelial cells were transfected with an shRNA to knock down NEXN-AS1 or a scramble (control) shRNA, followed by immunoblot analysis of the NEXN protein. Left: representative immunoblot images; right: fold differences in mean \pm SD NEXN band intensity after standardization against the band intensity of the housekeeping protein $\beta$-actin in 5 independent experiments. ${ }^{*} P<0.05, t$ test. (D) Cultured human vascular endothelial cells were subjected to ChIRP assay using NEXN-AS1 RNA probes (Supplemental Figure 3) to pull down NEXN-AS1 or probes for the LacZ gene as a control, followed by a PCR analysis using primers complementary to the NEXN gene 5 ' flanking region (Supplemental Figure 3). The graph shows fold difference in mean \pm SD NEXN PCR band intensity after standardization against the PCR band intensity of the housekeeping gene GAPDH in 5 replicate samples. ${ }^{*} P<0.05, t$ test. (E) Cultured vascular endothelial cells (HUVECs) were transfected with either a plasmid containing the firefly luciferase reporter gene and the NEXN gene $5^{\prime}$ flanking sequence (nucleotide positions -1245 bp to +297 bp relative to the transcription start site) with or without a deletion from +1 bp to +245 bp, together with a plasmid containing the Renilla luciferase gene to serve as a reference for transfection efficiency, with or without cotransfection of a lentivirus to overexpress NEXN-AS1, followed by measurement of firefly luciferase activity and Renilla luciferase activity. Column chart shows fold differences in mean \pm SD firefly luciferase activity after standardization against Renilla luciferase activity in 5 independent experiments. ${ }^{*} P<0.05, t$ test.

ticular interest, as it is known to be a regulator of gene transcription (12). To confirm that NEXN-AS1 interacts with BAZ1A, we carried out RNA immunoprecipitation using an anti-BAZ1A antibody, followed by RT-PCR detection of NEXN-AS1. The experiment demonstrated the presence of $N E X N-A S 1$ in the anti-BAZ1A antibody immunoprecipitate (Figure $3 \mathrm{~A}$ ), confirming an interaction between $N E X N-A S 1$ and BAZ1A. To determine which region of NEXN-AS1 interacts with BAZ1A, we performed pull-down experiments using RNA probes corresponding to the full-length NEXN-AS1 (nucle- otides 1-2292), the $5^{\prime}$ portion of NEXN-AS1 (nucleotides 1-1000), and the $3^{\prime}$ portion of NEXN-AS1 (nucleotides 1001-2292), respectively, followed by immunoblotting analysis using an anti-BAZ1A antibody. The analysis showed that the BAZ1A protein was present in the samples pulled down by either the full-length NEXN-AS1 probe or the probe corresponding to the 5' portion of NEXN-AS1, but dramatically diminished in the sample pulled down using the probe corresponding to the $3^{\prime}$ portion of NEXN-AS1 (Figure 3B), suggesting that BAZ1A primarily interacts with the 5 ' portion of NEXN-AS1. 
A

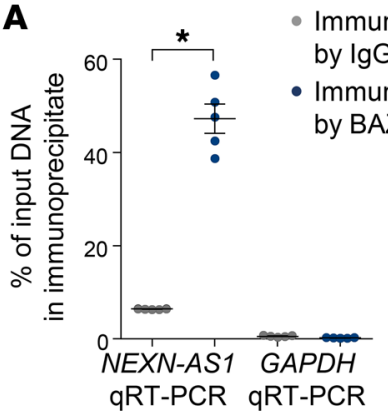

B
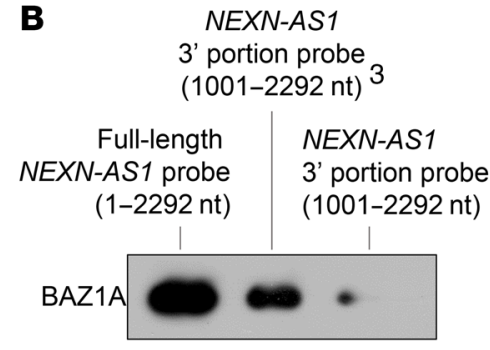

$\mathbf{E}$

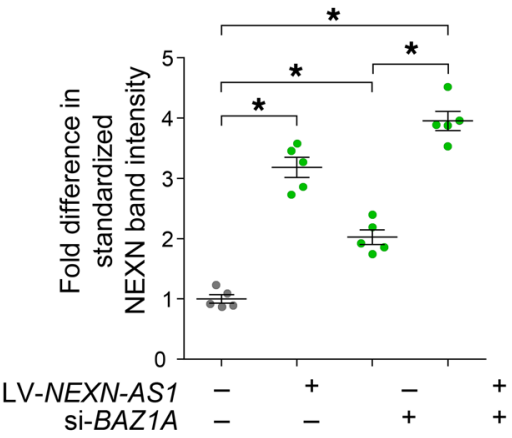

C

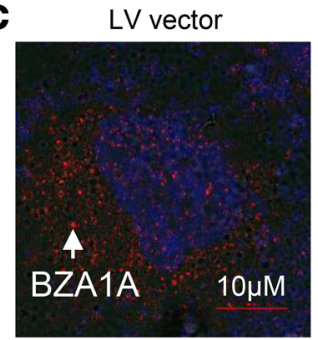

LV-NEXN-AS1

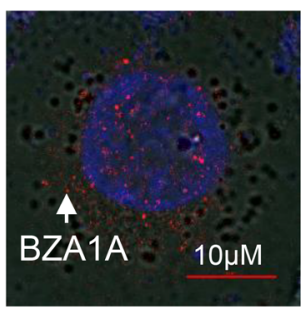

F

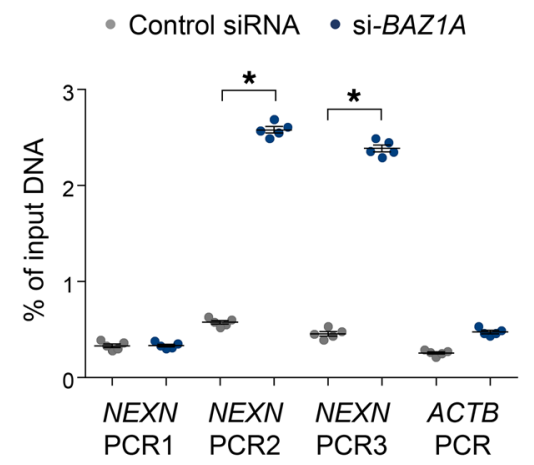

Figure 3. NEXN-AS1 interacts with BAZ1A, which downregulates NEXN expression. (A) Cultured human vascular endothelial cells were subjected to RNA immunoprecipitation using an anti-BAZ1A antibody, followed by quantitative RT-PCR analysis of NEXN-AS1. The graph shows fold differences in the amount of NEXN-AS1 (or the housekeeping gene GAPDH) in the immunoprecipitate captured by the anti-BAZ1A antibody versus the amount of NEXN-AS1 (or the housekeeping gene $G A P D H$ ) in the immunoprecipitate captured by an IgG isotope. Columns and error bars represent mean and SD values, respectiveIy, in 5 samples. ${ }^{*} P<0.05, t$ test. (B) Cultured vascular endothelial cell lysates were subjected to protein pulldown using either RNA probes corresponding to the full-length NEXN-AS1 (nucleotides 1-2292), the 5' portion of NEXN-AS1 (nucleotides 1-1000), and the 3' portion of NEXN-AS1 (nucleotides 1001-2292), respectively, or a control RNA probe for the housekeeping gene GAPDH, followed by immunoblotting analysis using an anti-BAZ1A antibody. (C) Cultured human vascular endothelial cells were transfected with either an NEXN-AS1-expressing lentivirus (LV-NEXN-AS1) or the lentivirus vector (LV vector) as a control, followed by immunofluorescence microscopy. BAZ1A was stained red with a fluorescence-labeled anti-BAZ1A antibody, and the nucleus was stained blue with DAPI. Scale bars: $10 \mu \mathrm{m}$. (D) Cultured human vascular endothelial cells were subjected to ChIP using the anti-BAZ1A antibody, followed by quantitative PCR analysis using primers annealing to the NEXN $5^{\prime}$ flanking sequence (Supplemental Figure 3 ) and the housekeeping gene $C A P D H$ as a reference. Graph shows mean and SD values in 5 samples. ${ }^{*} P<0.05, t$ test. (E) Cultured human vascular endothelial cells were transfected with either an NEXN-AS1-expressing lentivirus (LV-NEXN-AS1) or the lentivirus vector (LV vector) to serve as a control or with either a BAZ1A siRNA or a scramble (control) siRNA, followed by immunoblotting analysis using an anti-NEXN antibody and an antibody against the housekeeping protein $\beta$-actin as a loading control. The graph shows fold difference in mean ( \pm SD) NEXN band intensity after standardization against the band intensity of the housekeeping protein $\beta$-actin in 5 independent experiments. ${ }^{*} P<0.05$, ANOVA with post hoc analysis and Bonferroni's correction. (F) Cultured human vascular endothelial cells transfected with either a BAZ1A siRNA or a scramble (control) siRNA were subjected to FAIRE, followed by quantitative PCR analysis using primers complementary to the NEXN gene $5^{\prime}$ flanking sequence (Supplemental Figure 3). The graph shows mean and SD values from 5 experiments. ${ }^{*} P<0.05, t$ test.

RT-PCR and immunoblot analyses showed that BAZ1A was expressed in the arterial wall and that its levels were higher in atherosclerotic plaques than in the healthy arterial tissues (Supplemental Figure 4). Furthermore, we found that augmented NEXNAS1 expression in cultured vascular endothelial cells, monocytes, and VSMCs (by transfecting cells with an NEXN-AS1-expressing lentivirus) led to a decrease in BAZ1A levels (Figure 3C and Supplemental Figure 5). Immunofluorescence analysis of BAZ1A in cultured vascular endothelial cells showed that it was located in both the cytoplasm and nucleus (Supplemental Figure 6).

To investigate whether BAZ1A binds to the NEXN gene, we performed ChIP using an anti-BAZ1A antibody, followed by quantitative PCR using primers complementary to the NEXN $5^{\prime}$ flanking sequence. The assay detected the presence of the NEXN $5^{\prime}$ flanking sequence in chromatin samples pulled down by the anti-BAZ1A antibody (Figure 3D), confirming binding of BAZ1A to the NEXN gene. To investigate whether BAZ1A has an effect on NEXN expression, we performed siRNA-mediated BAZ1A knockdown in cultured vascular endothelial cells (Supplemental Figure 7). The experiment showed that BAZ1A knockdown led to an increase in NEXN levels and that this effect was further enhanced by augmented expression of NEXN-AS1 (Figure 3E), indicating that BAZ1A downregulates $N E X N$ expression. Furthermore, formaldehyde-assisted isolation of regulatory elements (FAIRE) analysis revealed that BAZ1A knockdown resulted in an open chromatin state in the NEXN 5' flanking region (Figure 3F), suggesting that BAZ1A downregulates $N E X N$ expression by condensing the chromatin in this region.

NEXN-AS1 and NEXN suppress the TLR4/NF- $\kappa B$ pathway, chemokine/cytokine expression by endothelial cells, and monocyte adhesion to endothelial cells. Since the abovementioned analyses showed that 
A

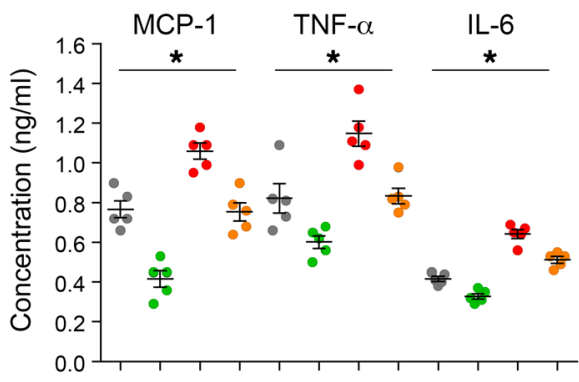

LV-NEXN-AS1 - + _ + _ + $+++_{+}++$ Si-NEXN - _ + + - _ + + - + +
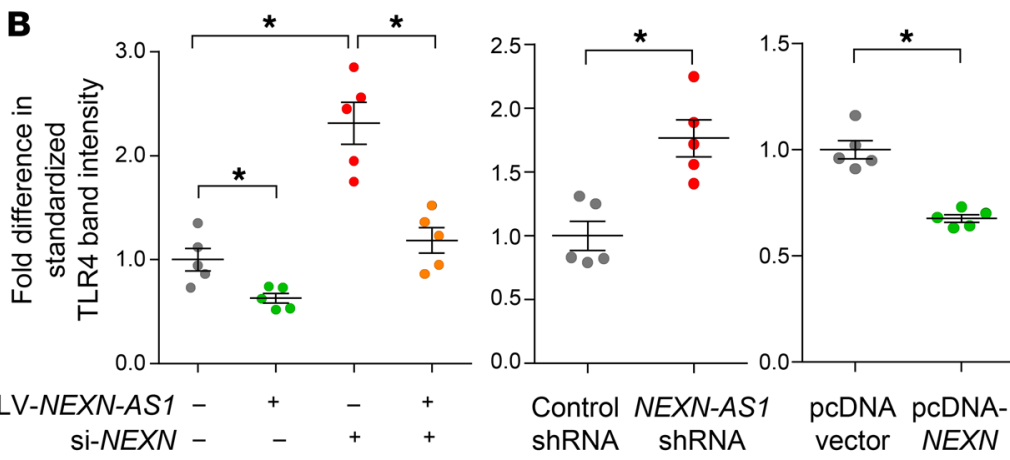

C

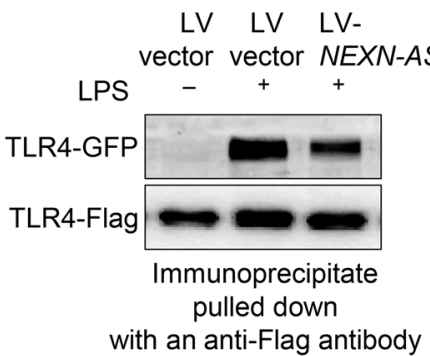

E

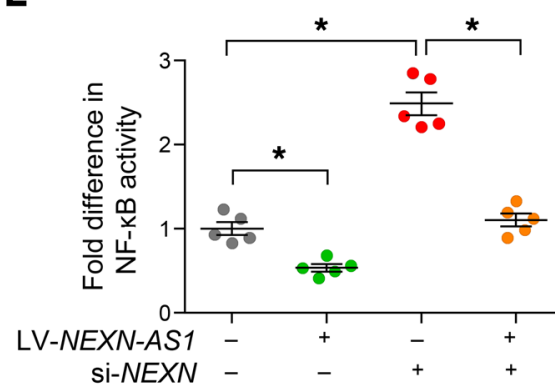

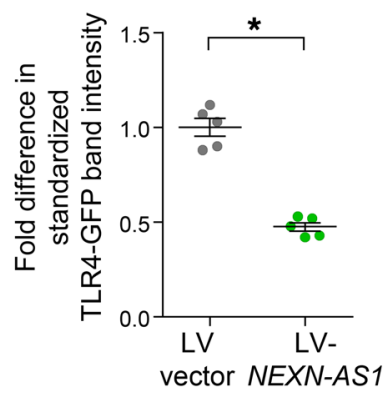

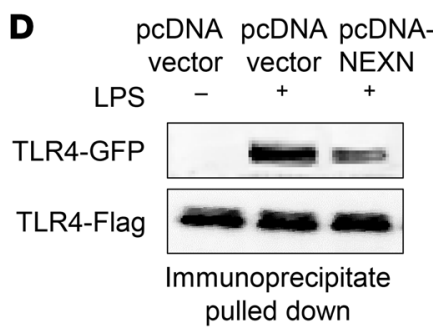

with an anti-Flag antibody
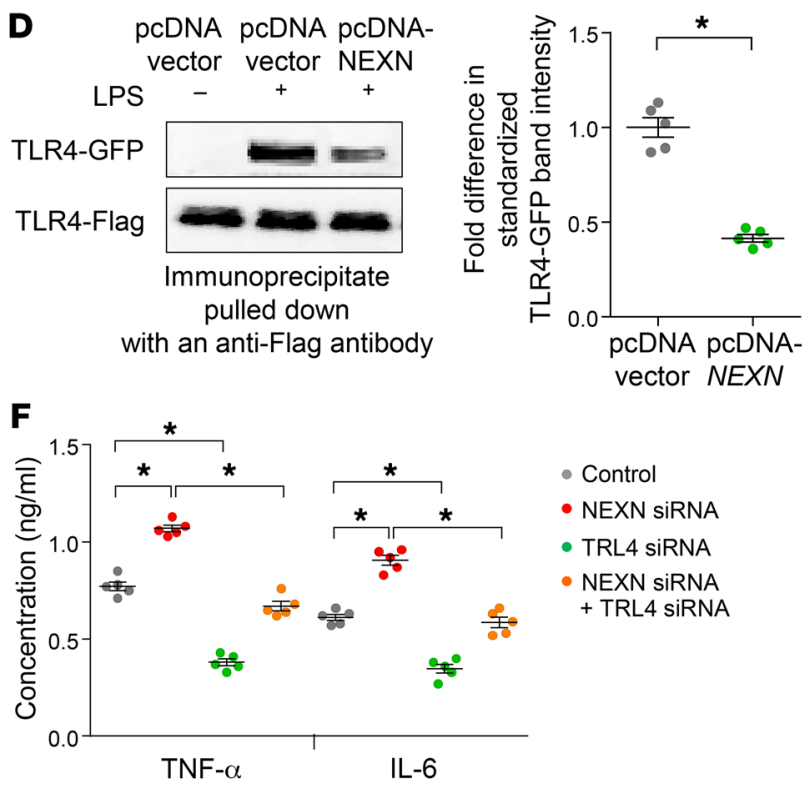

Figure 4. NEXN-AS1 and NEXN suppress the TLR4/NF-KB pathway and inflammatory gene expression. (A) Cultured human vascular endothelial cells were transfected with either an NEXN-AS1-expressing lentivirus (LV-NEXN-AS1), the lentivirus vector (LV vector), an NEXN siRNA, and/or a scramble (control) siRNA, then stimulated with lipopolysaccharides $(1 \mu \mathrm{g} / \mathrm{ml})$ for 12 hours, followed by enzyme-linked immunosorbent assay of MCP1, TNF- $\alpha$, and IL-6, respectively. (B) Cultured human vascular endothelial cells were transfected with either LV-NEXN-AS1, LV vector, an NEXN siRNA, scramble (control) siRNA, NEXNAS1 shRNA, scramble (control) shRNA, an NEXN-expressing plasmid (pcDNA-NEXN), and/or the plasmid vector (pcDNA-vector), followed by immunoblotting analysis using an anti-TLR4 antibody and an antibody against the housekeeping protein $\beta$-actin as a loading control. Graphs show fold differences in TLR4 band intensity standardized against $\beta$-actin band intensity. (C and D) Cells expressing FLAG-tagged TLR4 and GFP-tagged TLR4 were transfected with either LV-NEXN-AS1, LV vector, pcDNA-NEXN, or pcDNA-vector, and then stimulated with lipopolysaccharides $(1 \mu \mathrm{g} / \mathrm{ml})$ for 12 hours. Thereafter, cells were subjected to protein immunoprecipitation using anti-FLAG antibody, followed by immunoblotting analysis using either an anti-FLAG antibody or an antiGFP antibody. Representative immunoblot images are shown. Column charts show fold differences in TLR4-GFP band intensity standardized against TLR4Flag band intensity in 5 experiments. (E) Cultured human vascular endothelial cells were transfected with LV-NEXN-AS1, LV vector, NEXN siRNA, a scramble (control) siRNA, pcDNA-NEXN, and/or pcDNA vector, followed by NF- $\kappa B$ activity assay. (F) Cultured human vascular endothelial cells were transfected with NEXN siRNA, TLR4 siRNA, and/or a scramble (control) siRNA, followed by enzyme-linked immunosorbent assay of TNF- $\alpha$ and IL-6, respectively. Data are represented as mean \pm SD values from 5 independent experiments. ${ }^{*} P<0.05, t$ test or ANOVA with post hoc analysis and Bonferroni's correction.

NEXN-AS1 and NEXN are expressed in vascular endothelial cells, monocytes/macrophages, and VSMCs in atherosclerotic plaques, we investigated possible effects of NEXN-AS1 and NEXN on such cells.

We found that augmentation of NEXN-AS1 expression in cultured vascular endothelial cells reduced lipopolysaccharideinduced endothelial expression of the adhesion molecules ICAM1, VCAM1, and monocyte chemoattractant protein-1 (MCP1), the cytokines TNF- $\alpha$ and IL- 6 , and the extracellular matrix degrading enzymes MMP1 and MMP9 (Supplemental Figure 8). Furthermore, we found that knockdown of NEXN in endothelial cells resulted in increased MCP1, TNF- $\alpha$, and IL- 6 expression and abolished the inhibitory effect of NEXN-AS1 on MCP1, TNF- $\alpha$, and IL-6 expression (Figure 4A), indicating that $N E X N-A S 1$ exerts its effect on the expression of these molecules via NEXN.

Since the TLR4- and NF- $\kappa \mathrm{B}-$ mediated intracellular pathway plays a key role in the regulation of chemokine and cytokine expression $(13,14)$, we investigated a possible effect of NEXN-AS1 and NEXN on TLR4 and NF- $\kappa$ B activity. We found that augmentation of NEXN-AS1 expression suppressed lipopolysaccharide-induced increase of TLR4 levels in cultured vascular endothelial cells and 


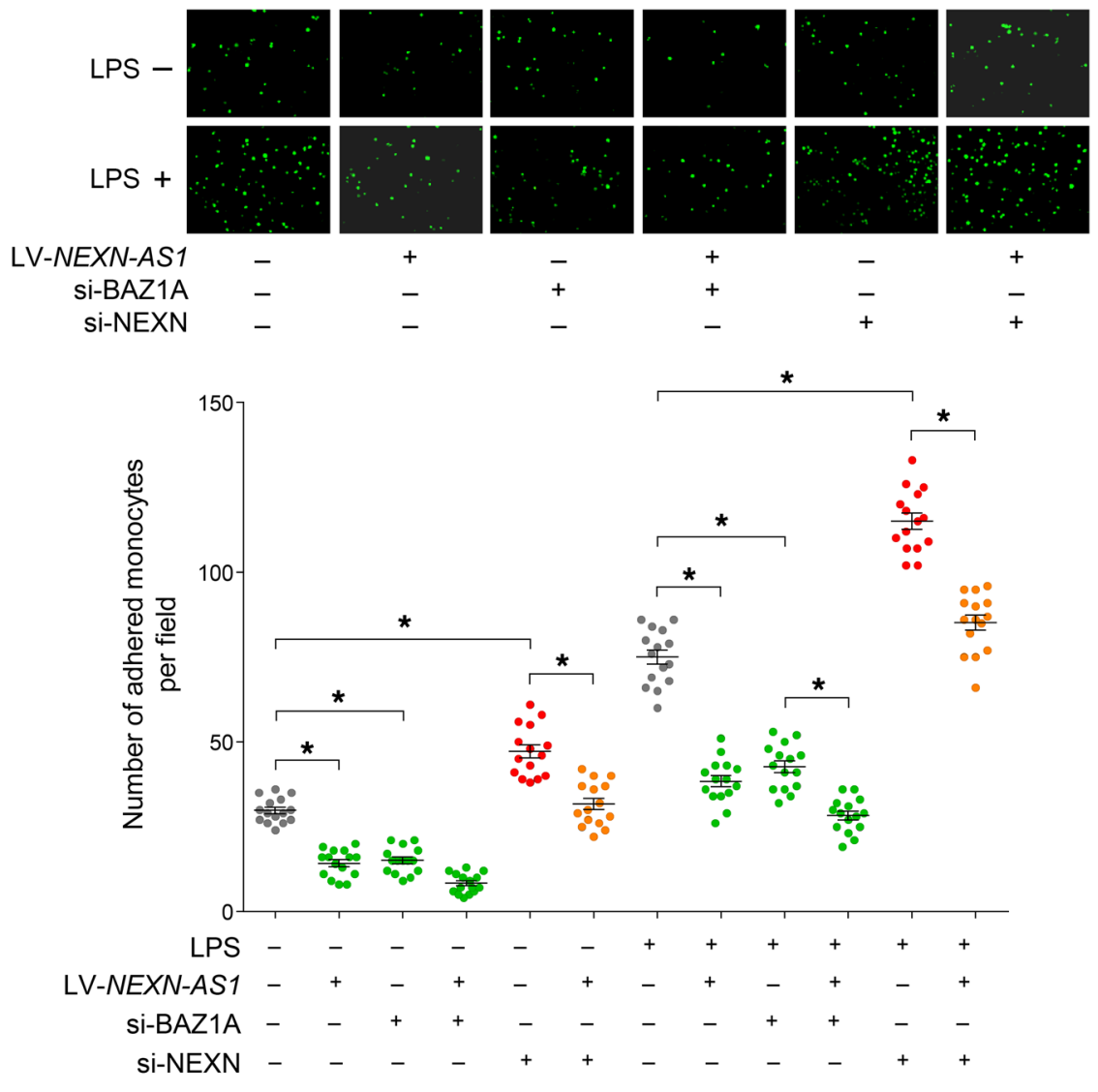

Figure 5. NEXN-AS1 and NEXN inhibit monocyte adhesion to endothelial cells. Cultured human vascular endothelial cells were transfected with either NEXN-AS1-expressing lentivirus (LV-NEXNAS1), BAZ1A siRNA (si-BAZ1A), or NEXN siRNA (si-NEXN), then either treated or not treated with lipopolysaccharides $(1 \mu \mathrm{g} / \mathrm{ml})$ for 4 hours and subsequently cocultured with fluorescently labeled monocytes for 1 hour, followed by fluorescent microscopy. Upper panel shows representative images of adhered monocytes. Original magnification, $\times 200$. The graph shows mean \pm SD number of adhered monocytes per microscopic field, from 5 independent experiments with each experiment performed in triplicate. ${ }^{*} P<0.05$, ANOVA with post hoc analysis and Bonferroni's correction.

that this inhibitory effect was abolished by NEXN knockdown (Figure $4 \mathrm{~B}$ and Supplemental Figures 7 and 9), while NEXN-AS1 knockdown led to an increase in TLR4 levels (Figure 4B). Additionally, we found that augmentation of NEXN-AS1 expression inhibited lipopolysaccharide-induced TLR4 oligomerization (Figure 4C). Furthermore, augmentation of $N E X N-A S 1$ expression in endothelial cells caused an increase in the levels of the NF- $\kappa B$ inhibitor I $\mathrm{B} \alpha \alpha$ in the cytoplasm (Supplemental Figure 10) and a decrease in NF- $\kappa \mathrm{B}$ DNA-binding activity in the nucleus (Figure 4E).

Analogous to overexpression of NEXN-AS1, augmentation of NEXN expression also reduced TLR4 levels and oligomerization and inhibited NF- $\kappa \mathrm{B}$ activity in cultured vascular endothelial cells (Figure 4, B, D and E, and Supplemental Figures 9 and 10). The inhibitory effect of increased NEXN-AS1 expression on NF- $\mathrm{NB}$ activity was abolished by NEXN knockdown (Figure 4E), indicating that the effect of $N E X N-A S 1$ on NF- $\kappa$ B activity was mediated by NEXN. Furthermore, NEXN knockdown in cultured vascular endothelial cells resulted in increased production of the inflammatory molecules TNF- $\alpha$ and IL-6, and this effect was abolished by TLR4 knockdown (Figure 4F), suggesting that NEXN exerts its effect on the production of these inflammatory molecules via the TLR4 pathway.

Moreover, we found that increased NEXN-AS1 expression in monocytes inhibited monocyte adhesion to vascular endothelial cells (Figure 5) and that knockdown of NEXN in monocytes had the opposite effect and abolished the inhibitory effect of NEXNAS1 overexpression on monocyte adhesion to endothelial cells (Figure 5). On the other hand, BAZ1A knockdown exerted an effect opposite of that of NEXN knockdown (Figure 5), in line with the finding from the experiments described earlier that BAZ1A downregulates NEXN expression.

Additionally, we observed that enhanced NEXN-AS1 expression in VSMCs reduced VSMC migration and that this inhibitory effect was abolished by NEXN knockdown in VSMCs (Supplemental Figure 11).

NEXN inhibits atherosclerotic lesion formation in ApoE $E^{-/-}$mice. To investigate whether NEXN plays a role in the pathogenesis of atherosclerosis, we crossbred $\mathrm{NEXN}^{+/-}$mice with $\mathrm{ApoE}^{-/-}$mice to generate $\mathrm{NEXN}^{+/-} \mathrm{ApoE}^{-/-}$mice $\left(\mathrm{NEXN}^{-/-} \mathrm{ApoE}^{-/-}\right.$mice could not be generated, likely due to being embryonic lethal). Both $N E X N^{+/-}$ ApoE ${ }^{-/-}$mice and $\mathrm{NEXN}^{+/+} \mathrm{ApoE}^{-/-}$littermates (controls) were fed a Western high-fat diet for 12 weeks. En face analysis of the aorta and cross-sectional analysis of the aortic root showed that $\mathrm{NEXN}^{+/-}$ $\mathrm{ApoE}^{-/-}$mice had substantially more atherosclerosis $(68.5 \%$ increase) than controls (Figure 6A), indicating that NEXN plays a protective role against atherogenesis.

Furthermore, histological and immunohistochemical analyses of aortic root cross sections showed that, compared with the atherosclerotic lesions in $\mathrm{NEXN}^{+/+} \mathrm{ApoE}^{-/-}$(control) mice, those in $\mathrm{NEXN}^{+/-}$ $A p o E^{-/-}$mice had reduced cap thickness (38.5\% lower), greater macrophage abundance (49.4\% higher), and higher lipid content $(44.3 \%$ higher), but lower VSMC and collagen content (39.5\% and $38.6 \%$ lower, respectively) and less calcification (77.5\% lower) (Figure 6A), with a higher vulnerability index $(1.46 \pm 0.31$ compared with $3.49 \pm$ 0.58 in controls, $P<0.001$ ), suggesting a protective role of NEXN against the development of vulnerable atherosclerotic plaques. 
A $-N E X N^{+/+} / A p o E^{-/-} \bullet N E X N^{+1-\mid} A p o E^{-1-}$
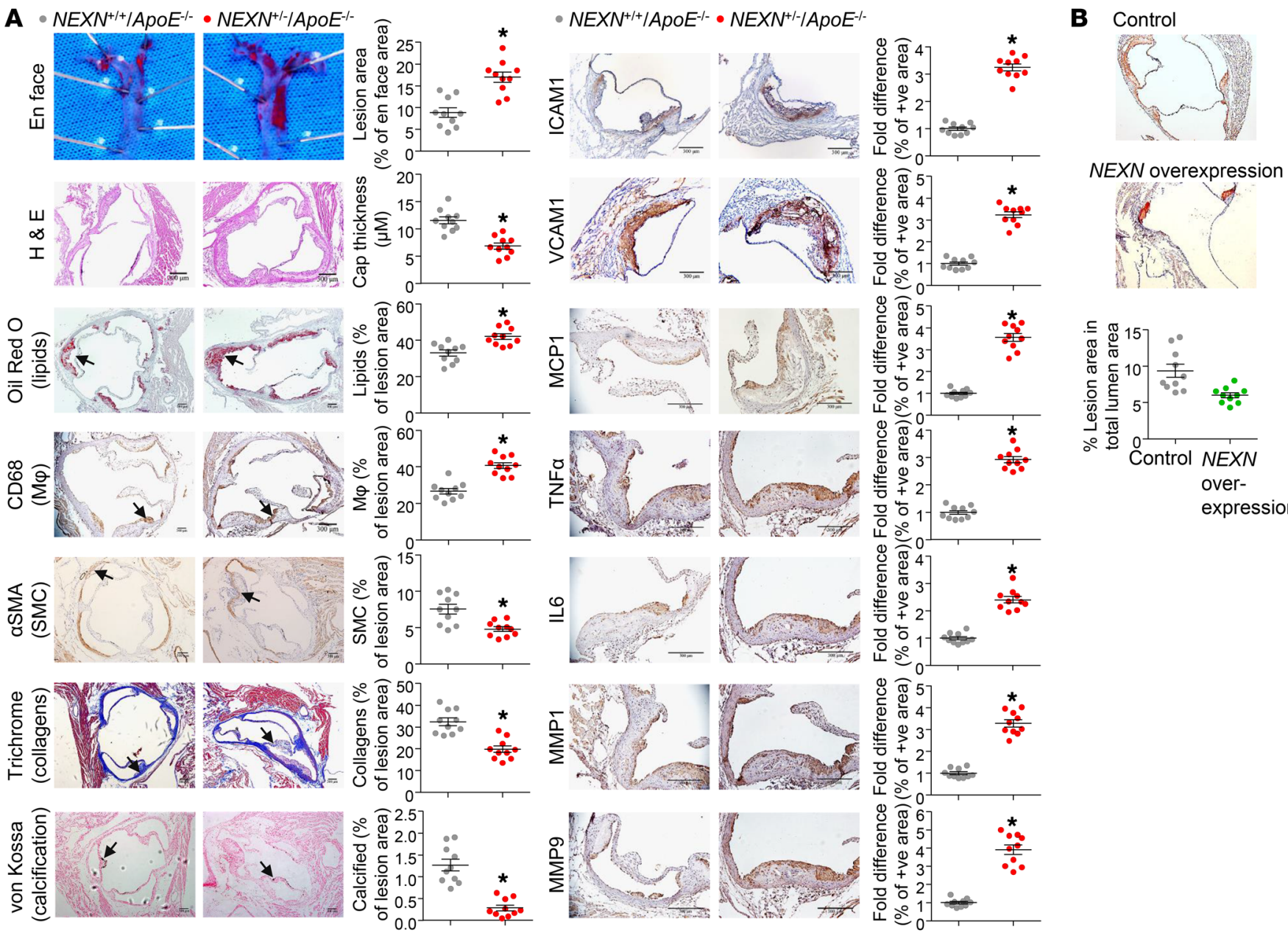

Figure 6. NEXN deters atherosclerosis in a mouse model. (A) $N E X N^{+/-} A p o E^{-/-}$mice and $N E X N^{+/+} A p o E^{-/-}$littermates, 6 weeks of age, were fed a Western high-fat diet for 12 weeks, followed by aorta en face oil red 0 staining and immunohistochemical analyses of aortic root cross sections. Shown in the figure are representative images of aorta en face oil red $\mathrm{O}$ staining and aortic root cross-section staining with $\mathrm{H} \& \mathrm{E}$, oil red $\mathrm{O}$ (for lipids), anti-CD68 antibody (for macrophages), anti- $\alpha$ SMA antibody (for VSMCs), trichrome (for collagens), von Kossa (for calcification), and antibodies against ICAM1, VCAM1, MCP1, TNF- $\alpha$, IL-6, MMP1, and MMP9, respectively. Scale bars: 300 uM. Graphs show differences (mean \pm SD) between the 2 groups in lesion area, cap thickness, and percentages of positive staining areas, respectively. $n=10$ animals in each group. ${ }^{*} P<0.05, t$ test. (B) ApoE ${ }^{-/-}$mice were injected with an adeno-associated virus to overexpress NEXN or with a negative control adeno-associated virus and fed the Western high-fat diet for 12 weeks, followed by oil red 0 staining of aortic root cross sections. Figure shows representative images of oil red 0 staining and mean \pm SD percentage of the atherosclerotic lesion area in total lumen area in the 2 groups. Original magnification, $\times 100$ (upper); $\times 200$ (lower). $n=10$ animals in each group. ${ }^{*} P<0.05, t$ test.

Further analyses showed that atherosclerotic lesions in $\mathrm{NEXN}^{+/} \mathrm{ApoE}^{-/-}$mice had higher levels of TLR4 (Supplemental Figure 12) and increased expression of adhesion molecules (ICAM1, VCAM1, and MCP1), inflammatory cytokines (TNF- $\alpha$ and IL-6), and matrix metalloproteinases (MMP1 and MMP9) than those in $\mathrm{NEXN}^{+/+} \mathrm{ApoE}^{-/-}$mice (Figure 6A), which may contribute to the protective role of NEXN against atherogenesis and plaque vulnerability and is in line with the aforedescribed results of assays of cultured human cells with NEXN knockdown.

To further test the effect of NEXN on atherogenesis, we injected $A p o E^{-/-}$mice with an adeno-associated virus to overexpress NEXN or with a negative control adeno-associated virus and fed the mice a Western high-fat diet for 12 weeks. The experiment showed that mice with NEXN overexpression had smaller atherosclerotic plaques than control mice (Figure 6B), further demonstrating that NEXN has a protective effect against atherosclerosis.
Circulating NEXN levels are lower in patients with CAD. Coronary artery atherosclerosis is the primary pathological cause of CAD, which can lead to myocardial infarction and heart failure. Having found a protective role of NEXN against atherosclerosis, we investigated whether there were differences in NEXN levels between individuals with and without CAD. We measured NEXN levels in blood serum samples from patients with CAD $(n=113)$, patients with myocardial infarction $(n=69)$, patients with heart failure ( $n=41$ ), and healthy individuals without any of these conditions $(n=40)$, by enzyme-linked immunosorbent assay. The assay showed that patients with $\mathrm{CAD}$, myocardial infarction, or heart failure had lower NEXN levels than healthy subjects $(290.57 \pm 152.12 \mathrm{pg} / \mathrm{ml}$, $305.56 \pm 137.68 \mathrm{pg} / \mathrm{ml}$ and $283.82 \pm 147.33 \mathrm{pg} / \mathrm{ml}$, respectively, compared with $393.65 \pm 113.87 \mathrm{pg} / \mathrm{ml}, P<0.05)$, in line with the finding that NEXN expression is lower in atherosclerotic plaques. Moreover, plasma NEXN levels were inversely correlated with plas- 
Table 1. Results of Pearson's correlation analysis of serum NEXN concentration in relation to biochemical measurements in the study subjects

\begin{tabular}{|c|c|c|c|c|c|c|c|c|}
\hline & \multicolumn{2}{|c|}{ Healthy controls $(n=40)$} & \multicolumn{2}{|c|}{$\operatorname{CCAD}(n=113)$} & \multicolumn{2}{|c|}{$\operatorname{AMI}(n=69)$} & \multicolumn{2}{|c|}{$\mathrm{HF}(n=41)$} \\
\hline & $r$ & $P$ value & $r$ & $P$ value & $r$ & $P$ value & $r$ & $P$ value \\
\hline Total cholesterol $(\mathrm{mmol} / \mathrm{l})$ & 0.021 & 0.727 & 0.057 & 0.467 & 0.028 & 0.869 & 0.242 & 0.404 \\
\hline Low density lipoprotein (mmol/l) & 0.079 & 0.198 & 0.110 & 0.161 & -0.024 & 0.847 & 0.458 & 0.099 \\
\hline High density lipoprotein (mmol/l) & 0.179 & 0.738 & 0.017 & 0.824 & 0.146 & 0.252 & 0.074 & 0.801 \\
\hline Total triglyceride (mmol/l) & 0.047 & 0.618 & -0.013 & 0.871 & -0.260 & 0.833 & 0.059 & 0.842 \\
\hline $\operatorname{ApoA1}(\mathrm{g} / \mathrm{l})$ & 0.039 & 0.592 & 0.019 & 0.808 & 0.442 & 0.719 & 0.195 & 0.505 \\
\hline Homocysteine $(\mu \mathrm{mol} / \mathrm{l})$ & -0.238 & 0.052 & -0.375 & 0.001 & -0.391 & 0.017 & -0.227 & 0.435 \\
\hline Myoglobin (ng/ml) & 0.030 & 0.627 & 0.099 & 0.206 & -0.031 & 0.789 & -0.033 & 0.870 \\
\hline Troponin I (ng/ml) & -0.025 & 0.687 & -0.153 & 0.051 & -0.026 & 0.843 & 0.155 & 0.443 \\
\hline Creatine phosphokinase (IU/I) & 0.074 & 0.226 & -0.183 & 0.019 & -0.164 & 0.042 & -0.281 & 0.330 \\
\hline Creatine phosphokinase-MB fraction (IU/I) & 0.173 & 0.069 & -0.221 & 0.005 & -0.312 & 0.803 & -0.340 & 0.203 \\
\hline
\end{tabular}

CCAD, chronic CAD; AMI, acute myocardial infarction; HF, heart failure. Bold indicates statistical significance $(P<0.05)$.

ma levels of homocysteine $(r=-0.375, P=0.001)$, creatine kinase $(r=-0.183, P=0.019)$, creatinekinase-muscle/brain (creatinekinaseMB) $(r=-0.221, P=0.005)$, and aspartate transaminase $(r=-0.236$, $P=0.002)$ in patients with CAD and with levels of homocysteine $(r=-0.391, P=0.017)$ and creatine kinase $(r=-0.164, P=0.042)$ in patients with myocardial infarction (Table 1$)$.

\section{Discussion}

One of the key findings from our study is that human atherosclerotic plaques have reduced expression of the NEXN gene, which encodes the actin-binding protein NEXN, as well as reduced expression of the nearby gene $N E X N-A S 1$, which encodes a previously functionally uncharacterized lncRNA, NEXN-AS1. Our study suggests that $N E X N-A S 1$ physically interacts with and increases the transcriptional activity of the $5^{\prime}$ flanking region of the NEXN gene, thereby upregulating $N E X N$ expression in vascular endothelial cells. Additionally, we found that $N E X N-A S 1$ also modulates $N E X N$ expression in monocytes and VSMCs.

Furthermore, our study reveals that NEXN-AS1 interacts with the nuclear protein BAZ1A and that BAZ1A interacts with the $5^{\prime}$ flanking region of the NEXN gene, although the specific motifs within NEXN-AS1 and NEXN responsible for BAZ1A binding are unclear. BAZ1A constitutes the accessory subunit of the ATP-dependent chromatin assembly factor (ACF), which consists of 2 subunits, the ATPase subunit SNF-2h and the accessory submit BAZ1A (12). ACF is known to be a chromatin remodeler that condenses chromatin, thereby repressing gene expression $(12,15$, 16). BAZ1A has previously been shown to suppress the expression of a number of genes, including RANKL, IGFBP3, and IGF1 (17). In the present study, we found that BAZ1A knockdown resulted in an open chromatin state in the NEXN $5^{\prime}$ flanking region and increased NEXN expression, suggesting that BAZ1A downregulates NEXN expression by condensing the chromatin in this region. Additionally, our study indicates that $N E X N-A S 1$ reduces the level of BAZ1A, which might in part explain the upregulatory effect of NEXN-AS1 on NEXN expression, although how NEXNAS1 reduces the BAZ1A levels is unclear. Since BAZ1A is capable of regulating multiple genes, it is conceivable that NEXN may be only one of the target genes of the NEXN-AS1/BAZ1A complex.

At the cellular level, enhanced NEXN-AS1 expression causes reduced production of TNF- $\alpha, \mathrm{IL}-6$, and MCP1 by endothelial cells and decreased monocyte adhesion to endothelial cells, and these effects are abolished if NEXN is knocked down, indicating that $N E X N-A S 1$ exerts its effects exclusively or predominantly via NEXN.

As highlighted earlier, the ENCODE project has discovered the existence of a large number of lncRNAs in the human genome, and there has been accumulating evidence revealing that at least some of the lncRNAs play important roles in regulating gene expression and participate in physiological and pathological processes (5-7). Therefore, there has been vast interest in characterizing the biological functions of the various lncRNAs. For example, the robust association between genetic variation on chromosome 9p21 and CAD susceptibility, discovered by genome-wide association studies (18), is at least in part mediated by the lncRNA CDKN2B-AS1 (also known as ANRIL), whose gene is located at this genomic locus (19). Further examples of lncRNAs that have been implicated in cardiovascular diseases are NovLnc6, Mhrt, MALAT1, and Tie-1-AS (7). The findings from our study described above add to the increasing understanding of the diverse biological roles of the various lncRNAs and, specifically, provide insight into the function of the previously uncharacterized lncRNA NEXN-AS1, with particular relevance to atherosclerosis. Interestingly, a recent study reported an association between variation in the NEXN-AS1 gene and risk of lung cancer in Europeans (20), raising the possibility that this lncRNA might also be involved in tumorigenesis.

Another key finding of our study is that NEXN confers a protective effect against atherosclerosis. Prompted by our initial finding that human atherosclerotic plaques have decreased NEXN expres- 
sion levels, we investigated to determine whether NEXN deficiency has a causative effect on the development of atherosclerosis. In ApoE-knockout mice fed a Western high-fat diet, the most widely used animal model of atherosclerosis, we found that targeted deletion of the NEXN gene resulted in substantially increased atherosclerotic lesion areas, indicating a protective role of NEXN against atherogenesis. In support, we demonstrate that overexpression of $N E X N$ in ApoE-knockout mice deters atherosclerosis. Furthermore, we observed that the atherosclerotic lesions in NEXN-deficient mice had higher levels of adhesion molecules (ICAM1, VCAM1, and MCP1), cytokines (TNF- $\alpha$ and IL-6), and extracellular matrix degrading enzymes (MMP1 and MMP9) as well as greater macrophage abundance and lipid content, but lower VSMC and collagen contents and less calcification, all of which are hallmarks of vulnerable atherosclerotic plaques $(3,21,22)$. Consistent with, and in support of, the findings from mice, the experiments using cultured human cells showed that NEXN knockdown resulted in increased production of MCP1, TNF- $\alpha$, and IL- 6 by endothelial cells and enhanced monocyte adhesion to endothelial cells. Furthermore, we found that NEXN inhibited TLR4 and NF- $\mathrm{BB}$ activity and that TLR4 knockdown abolished the effect of NEXN on TNF- $\alpha$ and IL-6 production, which provides a further mechanistic link, as it is well established that activation of the TLR4/NF- $\mathrm{BB}$ signaling pathway induces the expression of inflammatory molecules, including MCP1, TNF- $\alpha$, and IL-6, and plays an important role in endothelial activation and monocyte recruitment in atherogenesis $(14,23)$.

Mutations in the NEXN gene have been associated with dilated cardiomyopathy and hypertrophic cardiomyopathy in humans $(8,9)$. Additionally, a recent study reported an association of variation in the NEXN gene with susceptibility to CAD in Han Chinese and showed that NEXN inhibited balloon injury-induced neointima formation in a rat model (10). To our knowledge, our study is the first to show a role of NEXN in the development of de novo atherosclerosis, demonstrating that NEXN inhibits TLR4 and $\mathrm{NF}-\kappa \mathrm{B}$ activity, reduces inflammatory molecule expression by endothelial cells, suppresses monocyte recruitment, and deters atherogenesis.

NEXN is known as a filamentous actin-binding protein (24). Actin-binding proteins not only control the actin cytoskeleton functions in cell contractility, motility, and division, etc., but also influence cell-cell and cell-extracellular matrix interactions in addition to mediating intracellular signaling via interactions with various ligands (25). Interestingly, 2 other actin-binding proteins, namely profilin1 and Drebrin, were reported to be involved in atherogenesis and neointima formation, respectively $(26,27)$. Thus, evidence is growing to indicate that actin-binding proteins in muscle and nonmuscle cells play important roles in the development of these vascular pathological conditions. The finding of our study examining the actin-binding protein NEXN provides evidence in this regard.

In line with the initial finding that the NEXN expression level is reduced in atherosclerotic plaques, our study shows that patients with CAD have lower NEXN levels in blood samples than healthy control subjects. Although NEXN was found to be located at cell-matrix adherens junctions (24), the presence of NEXN in blood serum samples suggests that it can be released from cells, with possible autocrine, paracrine, and/or endocrine effects.
Interestingly, another actin-binding protein, namely profilin-1, has recently been reported to show elevated levels in blood samples of patients with CAD (28).

The results of our study inspire several interesting issues that warrant future investigation. These include (a) which factors affect NEXN-AS1 expression, leading to its reduction in atherosclerotic plaques; (b) whether NEXN expression is influenced by other factors apart from NEXN-AS1 and BAZ1A; and (c) whether expressing human $N E X N-A S 1$ will reduce atherogenesis using a well-established mouse model. It is noteworthy that mice do not have NEXN-AS1 and therefore it is not possible to do NEXN-AS1knockout experiments in mice.

In summary, the results of our study indicate that the lncRNA $N E X N-A S 1$ upregulates NEXN expression, thereby inhibiting vascular endothelial cell activation and monocyte adhesion via the TLR4/NF- $\mathrm{KB}$-mediated pathway and consequently deterring atherogenesis (Supplemental Figure 13). These findings are relevant to enhancing our understanding of the pathogenesis of atherosclerosis and indicate that $N E X N-A S 1$ and NEXN represent potential therapeutic targets.

\section{Methods}

Subjects. Atherosclerotic arterial samples were collected from patients undergoing carotid endarterectomy or abdominal aorta surgery at Nanfang Hospital. The carotid and aortic samples were jointly analyzed. Arteries without macroscopic evidence of atherosclerosis were collected from individuals who died either in a road traffic accident or due to cerebral edema. The tissue samples collected were snap-frozen in liquid nitrogen before the analyses of this study.

Blood samples were obtained from patients with CAD $(n=113)$, acute myocardial infarction $(n=69)$, or heart failure $(n=41)$ as well as from healthy individuals without any of these conditions $(n=40)$. Exclusion criteria were diabetes, cancer, or infection. The blood samples were taken before any medications were given to the patients. Serum was prepared from the blood samples and stored at $-80^{\circ} \mathrm{C}$ before assays. Additionally, demographic data and biochemical test results at hospital admission were collected from the hospital records. Demographic, biochemical, and clinical characteristics of the study subjects are shown in Supplemental Table 4.

Animals. ApoE $E^{-/-}$mice were purchased from the Laboratory Animal Center of Peking University (Beijing, China). $N E X N^{+/-}$mice were obtained from the European Mouse Mutant Archive (EM:05494). All mice had the C57BL/6J background. To generate ApoE and NEXN double-deficient mice, $N E X N^{+/-}$mice were crossbred with $A p o E^{-/-}$ mice. Due to embryonic lethality of NEXN-knockout homozygosity $\left(\mathrm{NEXN}^{-/-}\right), \mathrm{NEXN}^{+/-} \mathrm{ApoE}^{-/-}$and $\mathrm{NEXN}^{+/+} \mathrm{ApoE}^{-/-}$mice were used in this study. Six-week-old mice from both groups ( 5 males and 5 females in each group) were fed a Western high-fat diet for 12 weeks. Thereafter, mice were fasted for 4 hours, then placed under general anesthesia, after which blood was collected from the retro-orbital venous plexus using a capillary tube. The mice were then sacrificed by cervical dislocation, and tissues were collected for analysis. In a separate experiment, $A p o E^{-/-}$mice (5 males and 5 females in each group) were injected with an adeno-associated virus to overexpress NEXN or with a negative control adeno-associated virus and fed the Western high-fat diet for 12 weeks; thereafter, mice were sacrificed and tissues collected for analysis. 
Cell culture. Human monocytic cells (THP-1, ATCC TIB-202), vascular endothelial cells (HUVECs, ATCC CRL-1730), and VSMCs (human aorta VSMCs, ATCC CRL-1999) were obtained from ATCC. THP-1 cells were cultured in RPMI 1640 medium with $10 \%$ FCS. HUVECs and VSMCs were cultured in DMEM with $10 \%$ FCS. All cells were incubated at $37^{\circ} \mathrm{C}$ in an atmosphere of $5 \% \mathrm{CO}_{2}$. Cells were seeded in 6- or 12-well plates or $60 \mathrm{~mm}$ dishes and grown to $60 \%-80 \%$ confluence before use.

lncRNA and coding RNA microarray analysis. Total RNA was extracted from either atherosclerotic plaque cap specimens (from 3 individuals) or normal arterial wall samples (from 3 individuals) with the use of TRIzol reagent (Invitrogen) according to the manufacturer's protocol. RNA purity was assessed by spectrophotometry using NanoDrop ND-1000, and RNA integrity was verified by agarose gel electrophoresis. For microarray analysis, the Agilent microarray platform (Agilent Technologies) was used. Sample preparation and microarray hybridization were performed based on the manufacturer's standard protocols with minor modifications. Briefly, mRNA was purified from total RNA after removal of rRNA using the mRNA-ONLY Eukaryotic mRNA Isolation Kit (Epicentre). Then each mRNA sample was amplified and transcribed into fluorescent cRNA along the entire length of the transcripts without $3^{\prime}$ bias utilizing a random priming method. The labeled cRNAs were hybridized onto the Human LncRNA Expression Microarray, version $3.0(8 \times 60 \mathrm{~K}$, Arraystar). After washing the slides, the arrays were scanned using Agilent Scanner G2505C. Agilent Feature Extraction software (version 11.0.1.1) was used to analyze the acquired array images. Quantile normalization and subsequent data processing were performed using the GeneSpring GX v11.5.1 software package (Agilent). After quantile normalization of the raw data, lncRNAs and coding RNAs from at least 3 out of 6 samples flagged as present or marginal (all targets value) were chosen for further data analysis. Differentially expressed lncRNAs and coding RNAs statistically different between the 2 groups were identified through volcano plot filtering. All original microarray data were deposited in the NCBI's Gene Expression Omnibus database (GEO GSE97210).

Quantitative RT-PCR analysis. Total RNA from cultured cells and tissues was extracted using TRIzol reagent (Invitrogen) and reverse transcribed. Real-time PCR was performed on the ABI 7500 Real-Time PCR System (Applied Biosystems) with SYBR Green detection chemistry (Takara Bio). All samples were assayed in triplicate. Data were analyzed using the $\Delta \Delta$ Ct method, with U6 RNA as a reference in the lncRNA analysis and GAPDH as a reference in the analysis of protein-coding genes.

Immunoblot analysis. Protein extracts of cells and tissues were subjected to $10 \%$ sodium dodecyl sulfate-polyacrylamide gel electrophoresis, followed by immunoblot analyses using antibodies against either NEXN (Abcam, catalog ab83746 and ab233235), BAZ1A (Abcam, catalog ab90321), TLR4 (Abcam, catalog ab13556 and ab13867), ІкB $\alpha$ (Abcam, catalog ab32518), ICAM1 (Abcam, catalog ab171123), VCAM1 (Abcam, catalog ab134047), MCP1 (Abcam, catalog ab9669), TNF- $\alpha$ (Abcam ab6671), IL-6 (Abcam, catalog ab6672), MMP1 (Abcam, catalog ab52631), MMP9 (Abcam, catalog ab38898), or $\beta$-actin (Abcam, catalog ab8227) and chemiluminescence detection kits (ECL Plus Western Blot Detection System, Amersham).

Histological and immunohistochemical analyses. Sections (4 $\mu \mathrm{m}$ thick) of formalin-fixed, paraffin-embedded human atherosclerotic plaques and normal arterial wall specimens, respectively, were subjected to immunohistochemical staining using an anti-NEXN antibody (Abcam, catalog ab83746 and ab233235) recognizing amino acids 200-250 of NEXN.
To examine the extent of aortic atherosclerotic lesions in the studied mice, the aorta was opened longitudinally along the ventral midline from the iliac arteries to the aortic root, pinned out flat on a blue surface, and stained en face with oil red O (Sigma-Aldrich). Images of stained aortas were captured using a Leica DMI6000 microscope. The oil red O-stained areas were quantified using Image-Pro Plus 6.0 (Media Cybernetics).

Formalin-fixed, paraffin-embedded sections (3 $\mu \mathrm{m}$ thick) of mouse hearts with aortic root were stained with $\mathrm{H} \& \mathrm{E}$, oil red $\mathrm{O}$, Masson's trichrome for collagen, and von Kossa for calcification, respectively, or immunohistochemically stained using antibodies for CD68 (for macrophages, Abcam, catalog ab125212), $\alpha$-SMA (for VSMCs, Abcam, catalog ab124964), ICAM1 (Abcam, catalog ab171123), VCAM-1 (Abcam, catalog ab134047), MCP1 (Abcam, catalog ab25124), TNF- $\alpha$ (Abcam, catalog ab6671), IL-6 (Abcam, catalog ab7737), MMP1 (Abcam, catalog ab137332), and MMP9 (Abcam, catalog ab38898), respectively. Images were acquired using an Olympus BX50 microscope with a digital color camera and analyzed using Optimus software (version 6.2). The vulnerability index was calculated by the following formula: positive-staining area of (macrophages + lipid)/positive-staining area of (VSMCs + collagen).

Immunohistofluorescence analyses. Sections ( $4 \mu \mathrm{m}$ thick) of formalin-fixed, paraffin-embedded human atherosclerotic plaques and normal arterial wall specimens, respectively, were incubated with a rabbit anti-human NEXN antibody (Abcam, catalog ab83746) together with a mouse antibody for either human CD34 (Abcam, catalog ab8536), human $\alpha$-SMA (Abcam, catalog ab7817), or human CD68 (Abcam, catalog ab955), followed by incubation with a Texas redconjugated anti-rabbit secondary antibody (Abcam, catalog ab6719) and an FITC-conjugated anti-mouse secondary antibody (Abcam, catalog ab6785). Nuclei were counterstained with DAPI. Images were acquired using an Olympus BX50 fluorescence microscope with a digital color camera and analyzed using Optimus software (version 6.2).

Fluorescence confocal microscopy. Human vascular endothelial cells (HUVECs) were seeded on coverslips, fixed with paraformaldehyde, permeabilized with $0.25 \%$ Triton X-100, and then incubated with a digoxin-labeled probe (Dig-5'-AAGATATGTAGTGGCTTGGCTT-3'-Dig) for detecting NEXN-AS1. Thereafter, cells were incubated with a DyLight 594-conjugated IgG fraction (Abcam, catalog ab96873) coupled with a monoclonal mouse anti-digoxin antibody (Abcam, catalog ab116590). In a separate experiment, fixed and permeabilized HUVECs were incubated with an anti-NEXN antibody (Abcam, catalog ab83746) and subsequently with a fluorophore-conjugated goat anti-rabbit antibody (Abcam, catalog ab6717). Nuclei were counterstained with DAPI. Cells were imaged using an Olympus FV1000 confocal laser scanning microscope.

FISH. Paraffin-embedded sections ( $4 \mu \mathrm{m}$ thick) of human atherosclerotic plaques were deparaffinized, dehydrated, and treated with $1 \mathrm{M}$ sodium thiocyanate. Thereafter, the sections were digested in a pepsin solution, fixed in $4 \%$ formaldehyde, dehydrated by immersion in $70 \%$, $85 \%$, and $100 \%$ ethanol sequentially, and air dried. Subsequently, the sections were incubated with a digoxin-labeled probe (Dig-5'-AAGATATGTAGTGGCTTGGCTT-3'-Dig) for detecting NEXN-AS1 and then with a DyLight 594-conjugated IgG fraction (Abcam, catalog ab96873) coupled with a monoclonal mouse anti-digoxin antibody (Abcam, catalog ab116590). Nuclei were counterstained with DAPI. The stained sections were examined under an Olympus 710 NLO microscope. A minimum of 100 nuclei were evaluated for each sample. 
NEXN-AS1 copy number analysis. Total RNA was isolated from cultured human vascular endothelial cells (HUVECs), human monocytes (THP-1), and human VSMCs (CRL-1999, ATCC), respectively, and reversed transcribed into cDNA, followed by quantitative PCR of NEXN$A S 1$. The copy numbers of NEXN-AS1 RNA per cell were calculated using a standard curve generated by quantitative PCR using known amounts of NEXN-AS1 cDNA as templates, the numbers of cell from which RNA samples were extracted, and the Ct values of the NEXN-AS1 RT-PCR.

Lentivirus construction and cell infection. The NEXN-AS1 CDNA was amplified by PCR and cloned into the pLOV-EF1a-PuroR-CMV-EGFP2A-3FLAG vector, and the correct sequence of the NEXN-AS1 gene in this construct was verified by sequencing. This construct (referred to as LV-NEXN-AS1 hereafter) and the vector (control vector) were used to transfect cultured human vascular endothelial cells (HUVECs), human monocytes (THP-1), and human VSMCs (CRL-1999, ATCC), respectively, at a multiplicity of infection of 100 (for THP-1 cells) or 20 (for HUVECs and VSMCs) transfecting units per cell in the presence of $8 \mathrm{mg} / \mathrm{ml}$ of polybrene. The cells were washed with fresh complete media after 24 hours. Efficient $N E X N-A S 1$ overexpression was verified by quantitative RT-PCR (Supplemental Figure 2).

Recombinant plasmid construction. A plasmid containing the fulllength NEXN cDNA was purchased from OriGene. The NEXN cDNA was amplified by PCR and subcloned into the pcDNA3.1(+) vector. The correct sequence of the NEXN cDNA in the recombinant plasmid was verified by sequencing and named pcDNA3.1-NEXN. The plasmid was used to transfect HUVECs using Lipofectamine 3000 transfection reagent (Invitrogen).

NEXN-AS1, NEXN, BAZ1A, or TLR4 knockdown. Three shRNAs targeting different regions of NEXN-AS1 (Supplemental Table 5) were constructed using a lentivirus vector (pLKD-CMV-EGFP-2A-PuroU6-shRNA). These and a control shRNA were used to infect cultured endothelial cells (HUVECs). The siRNAs against NEXN, BAZ1A, and TLR4 (Supplemental Table 5), respectively, and an irrelevant siRNA to serve as a negative control were synthesized. Cultured endothelial cells (HUVECs) were transfected with each of these siRNAs using Lipofectamine 3000 (Invitrogen).

ChIRP. Human vascular endothelial cells (HUVECs) were crosslinked in 3\% formaldehyde for 30 minutes, followed by quenching in $0.125 \mathrm{M}$ glycine for 5 minutes. Cells were then lysed in a lysis buffer containing protease inhibitors, phenylmethylsulfonyl fluoride, and SUPERase-in RNase inhibitor (Thermo Fisher Scientific), followed by sonication using a Bioruptor ultrasonicator (Diagenode) at $4^{\circ} \mathrm{C}$ for at least 30 minutes (30 seconds on, 45 seconds off; output $=7$ ) until the cell lysate was clear. Subsequently, the sample was incubated with biotinylated antisense DNA tiling probes for pulling down NEXN-AS1 (Supplemental Table 6 and Supplemental Figure 3) at $37^{\circ} \mathrm{C}$ for 4 hours with shaking and then with streptavidin magnetic beads (Dynabeads MyOne Streptavidin C1; Thermo Fisher Scientific) at $37^{\circ} \mathrm{C}$ for $30 \mathrm{~min}-$ utes with shaking to capture the NEXN-AS1 bound chromatin.

To detect the presence of NEXN sequences in the above captured chromatin complex, DNA was then eluted and subjected to PCRs using the following 3 pairs of primers, respectively, to amplify the $5^{\prime}$ flanking region of the NEXN gene: primer pair 1, 5'-CTGCCACTTCTTCTCCCTTG-3' (forward) and 5'-AGTTGCTTTGCTGTGGCTTT-3' (reverse); primer pair 2, 5'-GCCTTTTCAACATCCCTGAA-3' (forward) and 5'-AAAACCCGAAATGCACACTC-3' (reverse); and primer pair 3, 5'-GGAAACTTGCTGCTGGAGAC-3' (forward) and 5'-ACCGGCGAAGGTACTTCTTT-3' (reverse) (Supplemental Figure 3). We also used a pair of primers to amplify the housekeeping gene GAPDH: 5'-CGGCTACTAGCGGTTTTACG-3' and 5'-AAGAAGATGCGGCTGACTGT-3', followed by agarose gel electrophoresis of the PCR products.

To identify the proteins in the above captured chromatin complex, proteins were eluted into an elution buffer composed of $12.5 \mathrm{mM}$ biotin (Invitrogen), $7.5 \mathrm{mM}$ 4-(2-hydroxyethyl)-1-piperazineethanesulfonic acid ( $\mathrm{pH} 7.5$ ), $75 \mathrm{mM}$ sodium chloride, $1.5 \mathrm{mM}$ ethylenediaminetetraacetic acid, $0.15 \%$ sodium dodecyl sulfate, $0.075 \%$ sarkosyl, and $0.02 \%$ sodium deoxycholate. Trichloroacetic acid ( $25 \%$ of the total volume) was then added, and the proteins were precipitated at $4^{\circ} \mathrm{C}$ overnight. Proteins were then pelleted by centrifugation, washed with cold acetone, and air dried. Proteins in the pellets were immediately solubilized in Laemmli sample buffer (Invitrogen) and boiled at $95^{\circ} \mathrm{C}$ for 30 minutes with occasional mixing to reverse crosslinking. Subsequently, the proteins were separated by bis-Tris sodium-dodecyl-sulfate polyacrylamide gel electrophoresis and then subjected to mass spectrometry analyses for protein identification.

Luciferase report assay. The NEXN gene $5^{\prime}$ flanking sequence (nucleotide positions -1245 bp to +297 bp relative to the transcription start site) with or without a deletion from +1 bp to +245 bp was cloned into the pGL4.10 vector (Promega) containing the firefly luciferase reporter gene. Cultured vascular endothelial cells (HUVECs) were transfected with either of these plasmids (together with a plasmid containing the Renilla luciferase gene to serve as a reference for transfection efficiency), with or without cotransfection of a lentivirus to overexpress $N E X N-A S 1$, followed by measurement of firefly luciferase activity and Renilla luciferase activity.

RNA immunoprecipitation using anti-BAZ1A antibody. Nuclear extracts from cultured human vascular endothelial cells (HUVECs) were prepared and incubated with an anti-BAZ1A antibody (Abcam, catalog ab90321) or a nonspecific mouse IgG at $4^{\circ} \mathrm{C}$ overnight. Protein G Plus Protein A agarose beads (Calbiochem) were then added, and the mix was incubated at $4^{\circ} \mathrm{C}$ for 2 hours, followed by centrifugation to pellet the beads. After washing and elution, immunoprecipitated RNA was extracted with the use of TRIzol reagent (Takara Bio), and RT-PCR (forward primer: 5'-CCGCCAGAATTTGTCTGTCTC-3'; reverse primer: 5'-TGACTGGAAACTAGGACGGG-3'; Supplemental Figure 3) was then performed to detect NEXN-AS1 binding to immunoprecipitated BAZ1A, followed by agarose gel electrophoresis.

Protein pulldown using NEXN-AS1 RNA probes. Cultured vascular endothelial cell (HUVEC) lysates were treated with DNase I and then incubated with streptavidin magnetic bead-conjugated RNA probes corresponding to the full-length NEXN-AS1 (nucleotide 1-2292), the $5^{\prime}$ portion of NEXN-AS1 (nucleotide 1-1000), and the 3' portion of NEXN-AS1 (nucleotide 1001-2292), respectively, or the housekeeping gene GAPDH. The samples pulled down by the RNA probes were subjected to immunoblotting analysis using an anti-BAZ1A antibody (Abcam, catalog ab90321).

ChIP. Human vascular endothelial cells (HUVECs) were crosslinked by incubation in formaldehyde and then incubated with glycine to quench formaldehyde. ChIP was carried out using an anti-BAZ1A antibody (Abcam, catalog ab90321) and the Pierce Agarose ChIP kit (Thermo Fisher Scientific) according to the manufacturer's instructions. DNA in the chromatin immunoprecipitated by the anti-BAZ1A antibody or an isotope IgG (Abcam, catalog ab172730) to serve as a 
control was subjected to quantitative PCR analysis of the NEXN gene 5 ' flanking sequence (using the same primers as those used in the ChIRP experiment described above, Supplemental Figure 3) and the housekeeping gene $G A P D H$, respectively.

FAIRE. Human vascular endothelial cells (HUVECs) with or without siRNA-mediated BAZ1A knockdown were crosslinked with formaldehyde and resuspended in lysis buffer, then sonicated to shear DNA to lengths between $200 \mathrm{bp}$ and $800 \mathrm{bp}$. The DNA lysates was subjected to phenol/chloroform/isoamyl alcohol to extract the open chromatin. The samples were decrosslinked by heating, and the residual proteins were digested by proteinase $\mathrm{K}$. The resulting DNA samples were subjected to PCRs using the following 3 pairs of primers, respectively, to amplify the $5^{\prime}$ flanking region of the $N E X N$ gene: primer pair 1, 5'-AGCGAAGTGGGTTCTTCAAC-3' (forward) and 5'-TGGCCAACATGGTGAAACTC-3' (reverse); primer pair 2, 5'-ATGAAGTGTTCCCGGATTGC-3' (forward) and 5'-TTCCAGTCAAGAAGCCAAGC-3' (reverse); and primer pair 3, 5'-AATTCTGGCGGAAAGTTGGC-3' (forward) and 5'-AAAACACAAGGCCAGTTCCC-3' (reverse) (Supplemental Figure 3). We also used a pair of primers to amplify the housekeeping gene: ACTB: 5'-TGACAAGGACAGGGTCTTCC-3' and 5'-CACCGTCCGTTGTATGTCTG-3'.

Cell adhesion assay. Human vascular endothelial cells (HUVECs) grown to confluency in 96-well plates were stimulated with lipopolysaccharides $(1 \mu \mathrm{g} / \mathrm{ml})$ for 4 hours. Thereafter, cells were washed and then cocultured with $1 \times 10^{5}$ calcein-labeled monocytic cells (THP-1) in DMEM without any supplement for 1 hour. After washing, THP- 1 cells adhering to the HUVEC monolayer were examined under BZ-9000 fluorescent microscopy (KEYENCE) and were quantified based on fluorescence intensities measured by an EnVision 2104 microplate fluorimeter (PerkinElmer). The numbers of THP-1 cells adhering to the HUVEC monolayer in each well were calculated from a standard curve generated by determining the intensity of fluorescence produced by known numbers of calcein-labeled THP-1 cells.

Cell migration assay. Human VSMC migration activity was assayed using the Transwell (BD Biosciences) method. In brief, human aorta VSMC (ATCC CRL-1999) culture was placed in the upper compartment, and media 231 supplemented with Smooth Muscle Growth Supplement (Invitrogen) was placed in the lower compartment. Following incubation at $37^{\circ} \mathrm{C}$ for 24 hours, the insert was removed from the well, and cells on the upper surface of the filter were removed using a cotton swab. Cells on the lower surface of the filter were fixed with paraformaldehyde, stained with crystal violet staining reagent, and counted under a light microscope. On each filter, cells in 9 different high-power fields were counted.

Enzyme-linked immunosorbent assay. The concentrations of MCP1, TNF- $\alpha$, and IL- 6 in vascular endothelial cell (HUVEC) culture supernatants as well as NEXN levels in human serum samples were measured with the use of ELISA kits (Cloud-Clone Corp.) following the manufacturer's protocol.

$N F-\kappa B$ activity assay. NF- $\kappa$ B p50 DNA-binding activity in nuclear extracts of vascular endothelial cells (HUVECs) was analyzed using an NF- $\kappa$ B p50 Transcription Factor Assay Kit (Abcam) following the protocol provided with the kit.
TLR4 oligomerization assay. Ba/F3 cells expressing FLAGtagged TLR4 and GFP-tagged TLR4 were (29) transfected with the aforedescribed LV-NEXN-AS1 lentivirus or the aforementioned pcDNA3.1-NEXN plasmid. Protein extracts from transfected cells were immunoprecipitated with the use of a monoclonal anti-FLAG antibody (Sigma-Aldrich, catalog F7425). The immunocomplex was subjected to sodium dodecyl sulfate-polyacrylamide gel electrophoresis and electrotransferred to a polyvinylidene difluoride membrane. The membrane was immunoblotted with an anti-FLAG antibody (Sigma-Aldrich, catalog F2555) and an anti-GFP antibody (Molecular Probes, catalog PA5-22688), respectively.

Statistics. Data were analyzed using Statistical Package for the Social Sciences (SPSS), version 13.0, software. Data are presented as mean \pm SD or median (interquartile range) unless otherwise indicated. Continuous variables were analyzed by 1 -way ANOVA with post hoc analysis and Bonferroni's correction for multiple comparisons or by unpaired 2-tailed Student's $t$ test, with $P<0.05$ being considered significant. Pearson's or Spearman's correlation analysis was used to determine correlations between selected protein levels and biochemical parameters, with $P<0.05$ being considered significant.

Study approval. The study was approved by the Committee for Ethical Review of Research Involving Human Subjects, Southern Medical University. Informed consent was obtained from the participants or relatives of deceased individuals. All animal experiments conformed to the Guide for the Care and Use of Laboratory Animals (NIH publication no. 85-23, revised 1996).

\section{Author contributions}

YWH and SY designed the study. FXG, YJX, PL, ZFL, CMK, SGW, JJZ, XM, ZY, FCF, YRQ, BMX, LX, QW, LMW, and LD conducted the experiments and acquired data. YWH, LZ, QW, and JJZ analyzed data. SY, YWH, and JJZ wrote the manuscript. SY, YWH, DGM, NJS, TRW, and JHY revised the manuscript.

\section{Acknowledgments}

This work was supported by the National Natural Sciences Foundation of China (grant nos. 81871701, 81572051, 81500387, and 81370202), the Natural Science Fund of Guangdong (grant nos. 2017A030313535 and 2018A030313533), the Science and Technology Program of Guangzhou (grant nos. 201607010267 and 201707010156), and the British Heart Foundation (grant nos. RG/16/13/32609 and PG/16/9/31995). This work falls under the portfolio of research conducted within the NIHR Leicester Biomedical Research Centre.

Address correspondence to: Yan-Wei Hu, Laboratory Medicine Center, Nanfang Hospital, Southern Medical University, Guangzhou, Guangdong 510515, China. Phone: 86.20.62786962; Email: ywhu0618@163.com. Or to: Shu Ye, Department of Cardiovascular Sciences, University of Leicester, BHF Cardiovascular Research Centre, Glenfield General Hospital, Groby Road, Leicester LE3 9QP, United Kingdom. Phone: 44.0116.2044754; Email: sy127@leicester.ac.uk.

\footnotetext{
1. Ross R. Atherosclerosis--an inflammatory disease. $N$ Engl J Med.1999;340(2):115-126.

2. Libby P. Inflammation in atherosclerosis. Nature.
}

2002;420(6917):868-874.

3. Libby P. Molecular bases of the acute coronary syndromes. Circulation. 1995;91(11):2844-2850.
4. Lander ES. Initial impact of the sequencing of the human genome. Nature. 2011;470(7333):187-197.

5. ENCODE Project Consortium. An integrated 
encyclopedia of DNA elements in the human genome. Nature. 2012;489(7414):57-74.

6. Batista PJ, Chang HY. Long noncoding RNAs: cellular address codes in development and disease. Cell. 2013;152(6):1298-1307.

7. Uchida S, Dimmeler S. Long noncoding RNAs in cardiovascular diseases. Circ Res. 2015;116(4):737-750.

8. Hassel D, et al. Nexilin mutations destabilize cardiac Z-disks and lead to dilated cardiomyopathy. Nat Med. 2009;15(11):1281-1288.

9. Wang $\mathrm{H}$, et al. Mutations in NEXN, a Z-disc gene, are associated with hypertrophic cardiomyopathy. Am J Hum Genet. 2010;87(5):687-693.

10. Wu C, et al. NEXN is a novel susceptibility gene for coronary artery disease in Han Chinese. PLOS ONE. 2013;8(12):e82135.

11. Stary HC. Natural history and histological classification of atherosclerotic lesions: an update. Arterioscler Thromb Vasc Biol. 2000;20(5):1177-1178.

12. Racki LR, et al. The chromatin remodeller ACF acts as a dimeric motor to space nucleosomes. Nature. 2009;462(7276):1016-1021.

13. Kawai T, Akira S. The role of pattern-recognition receptors in innate immunity: update on Toll-like receptors. Nat Immunol. 2010;11(5):373-384.

14. Hennessy EJ, Parker AE, O'Neill LA. Targeting Toll-like receptors: emerging therapeutics? Nat
Rev Drug Discov. 2010;9(4):293-307.

15. Fyodorov DV, Kadonaga JT. Dynamics of ATP-dependent chromatin assembly by ACF. Nature. 2002;418(6900):897-900.

16. Fyodorov DV, Blower MD, Karpen GH, Kadonaga JT. Acf1 confers unique activities to ACF/ CHRAC and promotes the formation rather than disruption of chromatin in vivo. Genes Dev. 2004;18(2):170-183.

17. Ewing AK, Attner M, Chakravarti D. Novel regulatory role for human Acf1 in transcriptional repression of vitamin D3 receptor-regulated genes. Mol Endocrinol. 2007;21(8):1791-1806.

18. Samani NJ, et al. Genomewide association analysis of coronary artery disease. $\mathrm{NEnglJ} \mathrm{Med.}$ 2007;357(5):443-453.

19. Holdt LM, et al. Circular non-coding RNA ANRIL modulates ribosomal RNA maturation and atherosclerosis in humans. Nat Commun. 2016;7:12429.

20. Yuan $\mathrm{H}$, et al. a novel genetic variant in long non-coding RNA gene NEXN-AS1 is associated with risk of lung cancer. Sci Rep. 2016;6:34234.

21. Davies MJ. Stability and instability: two faces of coronary atherosclerosis. The Paul Dudley White Lecture 1995. Circulation. 1996;94(8):2013-2020.

22. Wong KK, Thavornpattanapong P, Cheung SC, Sun Z, Tu J. Effect of calcification on the mechanical stability of plaque based on a three-dimen- sional carotid bifurcation model. BMC Cardiovasc Disord. 2012;12:7.

23. Michelsen KS, et al. Lack of Toll-like receptor 4 or myeloid differentiation factor 88 reduces atherosclerosis and alters plaque phenotype in mice deficient in apolipoprotein E. Proc Natl Acad Sci U S A. 2004;101(29):10679-10684.

24. Ohtsuka T, et al. Nexilin: a novel actin filamentbinding protein localized at cell-matrix adherens junction.JCell Biol.1998;143(5):1227-1238.

25. Olson EN, Nordheim A. Linking actin dynamics and gene transcription to drive cellular motile functions. Nat Rev Mol Cell Biol. 2010;11(5):353-365.

26. Romeo GR, Moulton KS, Kazlauskas A. Attenuated expression of profilin- 1 confers protection from atherosclerosis in the LDL receptor null mouse. Circ Res. 2007;101(4):357-367.

27. Stiber JA, et al. The actin-binding protein drebrin inhibits neointimal hyperplasia. Arterioscler Thromb Vasc Biol. 2016;36(5):984-993.

28. Caglayan E, et al. Profilin- 1 is expressed in human atherosclerotic plaques and induces atherogenic effects on vascular smooth muscle cells. PLOS ONE. 2010;5(10):e13608.

29. Youn HS, et al. Cinnamaldehyde suppresses tolllike receptor 4 activation mediated through the inhibition of receptor oligomerization. Biochem Pharmacol.2008;75(2):494-502. 